\title{
Intrauterine growth restriction alters growth performance, plasma hormones, and small intestinal microbial communities in growing-finishing pigs
}

Liang Xiong ${ }^{1,2}$, Jinming You ${ }^{2}$, Wanghong Zhang ${ }^{1}$, Qian Zhu' ${ }^{1}$, Francois Blachier ${ }^{3}$, Yulong Yin ${ }^{1}$ and Xiangfeng Kong ${ }^{1 *}$

\begin{abstract}
Background: The interaction of the gut microbiota with key metabolic and physiological processes may be associated with poor growth outcomes in animals born with intrauterine growth restriction (IUGR).

Results: Growth performance, plasma hormone concentrations, and intestinal microbiota composition were analyzed in IUGR pigs and in normal birth weight (NBW) pigs when the NBW pigs reached 25, 50, and $100 \mathrm{~kg}$ of body weight (BW). Compared to NBW pigs, IUGR pigs had lower initial, weaned, and final BW, and lower average daily gain and average daily feed intake in all the considered time points. In the $25 \mathrm{~kg}$ BW group, IUGR pigs had higher concentrations of plasma ghrelin and pancreatic polypeptide (PP), but lower insulin concentration than NBW pigs, while the situation was reversed in the $50 \mathrm{~kg}$ BW group. As compared to NBW pigs, IUGR pigs had higher microbial alpha diversity in the jejunum and ileum; in the 50 and $100 \mathrm{~kg}$ BW groups, IUGR pigs had higher Firmicutes abundance but lower Proteobacteria abundance in the jejunum, and lower Lactobacillus abundance in the jejunum and ileum; in the $25 \mathrm{~kg}$ BW group, IUGR pigs showed higher unclassified Ruminococcaceae abundance in the ileum; and in 25 and $50 \mathrm{~kg}$ BW groups, IUGR pigs showed lower Ochrobactrum abundance in the jejunum. Spearman's correlation revealed that Lactobacillus was negatively correlated with growth performance, while unclassified Ruminococcaceae was positively correlated. Predictive metagenomic analysis detected significantly different expression of genes in the intestinal microbiota between IUGR and NBW pigs, suggesting different metabolic capabilities between the two groups.

(Continued on next page)
\end{abstract}

\footnotetext{
* Correspondence: nnkxf@isa.ac.cn

${ }^{1}$ CAS Key Laboratory of Agro-ecological Processes in Subtropical Regions, Hunan Provincial Key Laboratory of Animal Nutritional Physiology and Metabolic Process, National Engineering Laboratory for Pollution Control and Waste Utilization in Livestock and Poultry Production, Institute of Subtropical Agriculture, Chinese Academy of Sciences, Changsha 410125, Hunan, China Full list of author information is available at the end of the article
}

C The Author(s). 2020 Open Access This article is licensed under a Creative Commons Attribution 4.0 International License, which permits use, sharing, adaptation, distribution and reproduction in any medium or format, as long as you give appropriate credit to the original author(s) and the source, provide a link to the Creative Commons licence, and indicate if changes were made. The images or other third party material in this article are included in the article's Creative Commons licence, unless indicated otherwise in a credit line to the material. If material is not included in the article's Creative Commons licence and your intended use is not permitted by statutory regulation or exceeds the permitted use, you will need to obtain permission directly from the copyright holder. To view a copy of this licence, visit http://creativecommons.org/licenses/by/4.0/ The Creative Commons Public Domain Dedication waiver (http://creativecommons.org/publicdomain/zero/1.0/) applies to the data made available in this article, unless otherwise stated in a credit line to the data. 
(Continued from previous page)

Conclusions: Growing-finishing IUGR pigs showed lower growth performance, higher microbial alpha diversity, and differences in plasma hormone concentrations compared to NBW pigs. Alterations in the abundance of Firmicutes, Proteobacteria, Ruminococcaceae, Lactobacillus, and Ochrobactrum in the small intestine may be associated with IUGR, and may therefore serve as a future target for gut microbiota intervention in growing-finishing IUGR pigs.

Keywords: Growing-finishing pigs, Growth performance, Intrauterine growth restriction, Microbial community, Small intestine

\section{Background}

Intrauterine growth restriction (IUGR), defined as the impaired growth and development of a mammalian embryo/fetus or fetal organs during pregnancy, is a major concern in pig farming [1]. Animals with IUGR are characterized by feeding intolerance, gut dysfunction, increased susceptibility to infection, and postnatal long-term growth limitation, resulting in higher morbidity and mortality early in their postnatal life [2]. IUGR occurs in 15$20 \%$ of newborn piglets and causes considerable economic losses in large-scale pig production farms [3]. Therefore, preventing IUGR and/or correcting its deleterious effects in growing animals is an important goal to improve the economic efficiency of pig production.

The small intestine, in addition to its role in digestion and nutrient absorption, is responsible for most immune system activities in mammals [4]. Previous studies confirmed that IUGR reduces the weight of the small intestine at birth and impairs its functions [5]. From a molecular perspective, IUGR is associated with modifications in the developmental pattern of the intestinal structure as well as with changes in the transcriptomic and proteomic profiles [6], which reduce the intestinal barrier function [7]. Therefore, the delay and alteration of gut development in piglets by IUGR are likely to play a major role in the slower growth rate [8].

The mammalian gastro-intestine harbors a large microbial community, the microbiota [9]. In pigs, the intestinal microbiota is involved in digestion and in the utilization of endogenous or acquired diet carbohydrates and proteins, production of vitamins, maintenance of intestinal morphology, regulation of immune responses, and establishment of the initial (innate) immune defense [10]. In addition, the intestinal microbiota synthesizes a large variety of metabolites, starting from dietary precursors, which are known to positively affect the energy metabolism and functions of the intestinal epithelial cells [11]. In addition, IUGR pigs are characterized by a different plasma concentration patterns for insulin, growth hormone, and insulin-like growth hormone, which can decisively affect the metabolic function, growth, and development of piglets $[12,13]$.

The existence of a link between intestinal microbiota and growth limitation in IUGR pigs is still unclear. In our previous study, the effects of IUGR on growth performance and intestine microbial community had been reported at 7,21 , and $28 \mathrm{~d}$ of age during the lactation [14]. Thus, the present study was conducted to determine the effects of IUGR on gut microbiota of growingfinishing pigs to investigate whether the subsequent performance are the carryover effect from the lactation. In the present study, we measured the differences in growth performance, concentration of plasma hormones, and small intestine microbiota profiles between IUGR and normal birth weight (NBW) pigs, to determine if IUGR is associated with long-lasting alterations in intestinal microbiota composition. A possible causal link between intestinal microbiota composition differences and changes in the physiological parameters is also discussed. The results obtained here could help identify plasma hormone- and intestinal microbiota-related biomarkers associated with growth performance in IUGR animals.

\section{Materials and methods}

\section{Experimental design, animals, and diets}

A total of 36 parity 3 and 4 sows (Large White and Landrace) from three farrowing groups (12 sows each group) were selected from an experimental herd located in Yong'an Town, Liuyang City, Hunan Province, China. After delivery, 72 castrated male piglets were obtained from a total of 36 litters with 10 to 12 born alive piglets, including one IUGR piglet and one NBW piglet per litter. Piglets with a birth weight greater than the mean birth weight per litter were classified as NBW piglets, while piglets with the lowest birth weight per litter were classified as IUGR piglets. Our study selected pregnant sows of three different farrowing groups at different periods and the piglets used in this study were from three farrowing groups for 25, 50, and $100 \mathrm{~kg}$ body weight (BW) groups. Thus, this study was divided into three independent trials.

Piglets were weaned at $27 \mathrm{~d}$ of age and transferred to a nursery facility. To reduce the influence of mutual attack and psychological stress of weaned piglets after commingling, the pigs were individually penned in this study. The piglets were housed in an environmentally controlled facility with hard plastic and slatted flooring. Each $0.6 \mathrm{~m} \times 1.2 \mathrm{~m}$ pen was equipped with a single-hole 
feeder and a water nipple to allow ad libitum consumption of feed (provided twice daily at 8:00 and 16:00) and drinking water. Therefore, the pigs were remained in nursery facility until they reached $165 \mathrm{~d}$ of age. A nursery diet was fed at $28-69 \mathrm{~d}$ of age, a growing diet at $70-103 \mathrm{~d}$ of age, and a finishing diet at 104-165 d of age (Table 1). The study was completed when average body weight of NBW pigs reached 25, 50, and $100 \mathrm{~kg}$ for 25 $\mathrm{kg} \mathrm{BW}, 50 \mathrm{~kg} \mathrm{BW}$, and $100 \mathrm{~kg}$ BW groups, respectively. Pigs were fed in three phases feeding regime which diets were offered to the pigs as pellets for $25 \mathrm{~kg}, 50 \mathrm{~kg}$, and $100 \mathrm{~kg} \mathrm{BW}$ groups, respectively. The change of phases was made when the average BW of NBW pigs for $25 \mathrm{~kg}$, $50 \mathrm{~kg}$, and $100 \mathrm{~kg} \mathrm{BW}$ groups reached $25 \mathrm{~kg}, 50 \mathrm{~kg}$, and $100 \mathrm{~kg}$. The dietary nutrient levels were referred to NRC (2012) recommendation and considered the commercial pig production. The diets used in this study were provided by the pig farm. No antibiotics were used during the entire study. The NBW and IUGR piglets from each litter had the same source of breast-milk, farrowing pens, and growth environment during lactation, to ensure that the initial colonization of intestinal microbiota was similar.

\section{Growth performance}

Each pig was weighed at birth (initial BW), at weaning (27 days old) and at the end of each stage (including nursery, growing, and finishing), to calculate average daily gain (ADG). Feed intake and remaining feed per pen were recorded weekly and at the end of each stage to calculate the average daily feed intake (ADFI) and feed/gain $(F / G)$ ratio.

\section{Sample collection}

When the average BW of NBW pigs reached 25, 50, and $100 \mathrm{~kg} \mathrm{BW}, 10$ pigs per group were randomly weighed

Table 1 Composition and nutrient levels of experimental diets (as air-dried)

\begin{tabular}{|c|c|c|c|}
\hline Items & Nursery pig feed (28-69 d of age) & Growing pig feed (70-103 d of age) & Finishing pig feed (104-165 d of age) \\
\hline \multicolumn{4}{|l|}{ Ingredients, \% } \\
\hline Corn & 60.00 & 61.00 & 61.17 \\
\hline Barley & 6.00 & 8.00 & 8.00 \\
\hline Soybean oil & 2.00 & 1.50 & 1.00 \\
\hline Soybean meal & 27.50 & 25.00 & 25.50 \\
\hline $\mathrm{CaHPO}_{4}$ & 0.10 & 0.10 & 0 \\
\hline Lysine & 0.16 & 0.18 & 0.13 \\
\hline Methionine & 0.02 & 0.03 & 0.00 \\
\hline Threonine & 0.10 & 0.07 & 0.08 \\
\hline Anti-oxidant & 0.02 & 0.02 & 0.02 \\
\hline Anti-mildew agent & 0.10 & 0.10 & 0.10 \\
\hline Nursery pigs premix ${ }^{a}$ & 4.00 & 0 & 0 \\
\hline Growing-finishing pigs premix ${ }^{b}$ & 0 & 4.00 & 4.00 \\
\hline Total & 100.00 & 100.00 & 100.00 \\
\hline \multicolumn{4}{|l|}{ Nutrient levels ${ }^{c}$} \\
\hline Digestible energy, MJ/kg & 13.91 & 13.77 & 13.64 \\
\hline Crude protein & 17.20 & 16.40 & 16.50 \\
\hline Crude fat & 4.70 & 4.30 & 3.80 \\
\hline Crude fiber & 2.70 & 2.70 & 2.80 \\
\hline Digestible lysine & 1.17 & 1.08 & 1.05 \\
\hline Digestible methionine & 0.33 & 0.30 & 0.28 \\
\hline Digestible threonine & 0.77 & 0.71 & 0.73 \\
\hline Total calcium & 0.77 & 0.74 & 0.66 \\
\hline Total phosphorus & 0.56 & 0.52 & 0.45 \\
\hline
\end{tabular}

${ }^{a}$ Nursery pig premix supplied per kg feed: $8,000 \mathrm{IU}$ vitamin $\mathrm{A}, 228 \mathrm{IU}$ vitamin $\mathrm{D}_{3}, 15 \mathrm{IU}$ vitamin $\mathrm{E}, 3.0 \mathrm{mg}$ vitamin $\mathrm{K}_{3}, 1.3 \mathrm{mg}$ vitamin $\mathrm{B}_{1}, 3.1 \mathrm{mg}$ vitamin $\mathrm{B}_{2}, 1.2 \mathrm{mg}$ vitamin $B_{6}, 0.03 \mathrm{mg}$ vitamin $B_{12}, 13.4 \mathrm{mg}$ calcium pantothenate, $500 \mathrm{mg}$ choline chloride, $120 \mathrm{mg}$ iron, $10 \mathrm{mg}$ copper, $130 \mathrm{mg}$ zinc, $100 \mathrm{mg}$ manganese, $0.3 \mathrm{mg}$ iodine, and $0.3 \mathrm{mg}$ selenium

${ }^{b}$ Growing-finishing pig premix supplied per kg feed: $15,000 \mathrm{IU}$ vitamin $\mathrm{A}, 200 \mathrm{IU}$ vitamin $\mathrm{D}_{3}, 50 \mathrm{IU}$ vitamin $\mathrm{E}, 4.0 \mathrm{mg}$ vitamin $\mathrm{K}_{3}, 4.0 \mathrm{mg}$ vitamin $\mathrm{B}_{1}, 10 \mathrm{mg}$ vitamin $B_{2}, 3.0 \mathrm{mg}$ vitamin $B_{6}, 0.04 \mathrm{mg}$ vitamin $B_{12}, 20.0 \mathrm{mg}$ calcium pantothenate, $800 \mathrm{mg}$ choline chloride, $120 \mathrm{mg}$ iron, $20 \mathrm{mg}$ copper, $112 \mathrm{mg}$ zinc, $124 \mathrm{mg}$ manganese, $0.5 \mathrm{mg}$ iodine, $0.4 \mathrm{mg}$ selenium

${ }^{c}$ Nutrient levels were calculated values 
and blood samples were collected from the precaval vein $12 \mathrm{~h}$ after the last feeding, for plasma hormone concentration determination. The sampled animals were exsanguinated after electrical stunning. The luminal contents of the jejunum $(10 \mathrm{~cm}$ below the flexura of duodenum-jejunum) and ileum $(10 \mathrm{~cm}$ above the ileocecal junction) were sampled and stored at $-80^{\circ} \mathrm{C}$ for microbiota composition analysis.

\section{Concentration of plasma hormones}

Plasma concentrations of gastrin, growth hormone $(\mathrm{GH})$, ghrelin, glucagon, insulin-like growth factor-1 (IGF-1), insulin, leptin, pancreatic polypeptide (PP), peptide YY (PYY), and somatostatin (SS) were assayed using the Meimian ELISA kit (Suzhou Yutong Biotechnology Company, Suzhou, Jiangsu, China), as per the manufacturer's instructions, and then read on a spectrophotometer (Biomate 5, Thermo Electron Corporation, Rochester, NY, USA).

\section{Microbiota DNA isolation and PCR amplification}

Total bacterial genomic DNA was extracted from intestinal samples using the Fast DNA SPIN extraction kit (MP Biomedicals, Santa Ana, CA, USA) following the manufacturer's instructions, and stored at $-20^{\circ} \mathrm{C}$ until further analysis. The quantity and quality of the extracted DNA were assessed using a NanoDrop ND-1000 spectrophotometer (Thermo Fisher Scientific, Waltham, MA, USA) and agarose gel electrophoresis, respectively.

The bacterial 16S rRNA gene V3-V4 region was amplified by PCR using the forward primer $338 \mathrm{~F}\left(5^{\prime}\right.$ ACTCCTACGGGAGGCAGCA-3') and the reverse primer 806R (5'-GGACTACHVGGGTWTCTAAT-3'). Sample-specific 7-bp barcodes were added to the primers for multiplex sequencing. The PCR mix included the following components: $5 \mu \mathrm{L}$ Q 5 reaction buffer $(5 \times), 5 \mu \mathrm{L}$ Q5 High-Fidelity GC buffer ( $5 \times), 0.25 \mu \mathrm{L}$ Q5 High-Fidelity DNA Polymerase $(5 \mathrm{U} / \mu \mathrm{L}), 2 \mu \mathrm{L}(2.5 \mathrm{mmol} / \mathrm{L})$ dNTPs, $1 \mu \mathrm{L}$ each $(10 \mu \mathrm{mol} / \mathrm{L})$ of forward and reverse primers, $2 \mu \mathrm{L}$ DNA template, and $8.75 \mu \mathrm{L}$ double distilled water. The Quant-iT ${ }^{\mathrm{mm}}$ PicoGreen $^{\mathrm{mm}}$ dsDNA Assay Kit used in the PCR assay was purchased from Thermo Fisher Scientific (USA). Amplification reactions were carried out with the following profile: $2 \mathrm{~min}$ of initial denaturation at $98^{\circ} \mathrm{C}, 25$ cycles of $15 \mathrm{~s}$ at $98^{\circ} \mathrm{C}, 30 \mathrm{~s}$ at $55^{\circ} \mathrm{C}$, and $30 \mathrm{~s}$ at $72{ }^{\circ} \mathrm{C}$, and final extension at $72{ }^{\circ} \mathrm{C}$ for $5 \mathrm{~min}$. Amplicons were further purified using Agencourt AMPure Beads (Beckman Coulter, Indianapolis, IN, USA) and quantified using the PicoGreen dsDNA assay kit (Invitrogen, Carlsbad, CA, USA) following the manufacturer's protocols. Purified amplicons were grouped in equimolar pools, and pairedend $(2 \times 300 \mathrm{bp})$ sequencing was performed using the MiSeq Reagent Kit v3 (600 cycles) on an Illumina MiSeq platform (Illumina, San Diego, CA, USA), according to the standard protocols established by Shanghai Personal Biotechnology Co. Ltd. (Shanghai, China).

\section{Statistical analyses}

Significance of the differences in growth performance and plasma hormone profile between IUGR and NBW pigs was assessed by the Student's $t$-test and for microbiota alpha diversity and abundance by the MannWhitney U-test. Both analyses were performed on SPSS 22.0 (Chicago, IL, USA). Alpha diversity (ACE, Chao1, Shannon, and Simpson indices) was assessed in QIIME 1.8 (http://qiime.org/). The beta diversity was assessed to investigate structural variation in the intestinal microbiota among samples by principal coordinate analysis (PCoA) based on unweighted UniFrac distance. Partial least squares discriminant analysis (PLS-DA) based on unweighted UniFrac distance with constrained ordination and supervised learning was also performed to reveal the intestinal microbiota variation among samples [15]. Linear discriminant analysis (LDA) effect size (LEfSe) and statistical analysis of metagenomic profiles (STAMP) 2.1.3 software were used to simultaneously compare differences in taxonomic levels, including phylum and genus, between IUGR and NBW pigs. Phylogenetic Investigation of Communities by Reconstruction of Unobserved States (PICRUSt) was used to characterize the functional capacity of the small intestine microbiota of growing-finishing pigs. In LEfSe analysis, the non-parametric factorial Kruskal-Wallis (KW) sumrank test was used to detect all species with significant differential abundance and the Wilcoxon rank-sum test was used to investigate biological consistency among subclasses [16]. As a last step, histograms of the LDA score were used to measure the effect size for determining the significantly different taxa and metabolic functions based on the observed relative differences. Spearman's correlation between growth performance and intestinal microbiota composition was calculated using the $\mathrm{R}$ package ggplot2 3.3.1 (https://www.r-project.org/). GraphPad Prism 6.0 (San Diego, CA, USA) was used to plot the images. Data are shown as means \pm SEM. Differences between IUGR pigs and NBW pigs were considered significant when the $P$-value $<0.05$.

\section{Results \\ Growth performance}

In the 25 and $50 \mathrm{~kg}$ BW groups, IUGR pigs had significantly lower initial BW, weaned BW, final BW, ADG, and ADFI $(P<0.05)$ than NBW pigs, but we observed no significant differences in F/G (Table 2). In the $100 \mathrm{~kg}$ BW group, IUGR pigs had significantly lower initial BW, weaned $\mathrm{BW}$, final $\mathrm{BW}$, and ADG than NBW pigs $(P<$ $0.05)$, but ADFI and F/G were not significantly different. 
Table 2 Effect of intrauterine growth restriction (IUGR) on growth performance of growing-finishing pigs

\begin{tabular}{|c|c|c|c|c|c|c|}
\hline \multirow[t]{2}{*}{ Items } & \multicolumn{2}{|c|}{$25 \mathrm{~kg}$ BW group } & \multicolumn{2}{|c|}{50 kg BW group } & \multicolumn{2}{|c|}{100 kg BW group } \\
\hline & NBW & IUGR & NBW & IUGR & NBW & IUGR \\
\hline Initial BW, kg & $1.77 \pm 0.09$ & $1.04 \pm 0.07^{* *}$ & $1.79 \pm 0.06$ & $1.01 \pm 0.03^{* *}$ & $1.86 \pm 0.10$ & $0.96 \pm 0.04^{* *}$ \\
\hline Weaned BW, kg & $10.39 \pm 0.37$ & $7.23 \pm 0.51^{* *}$ & $13.03 \pm 0.47$ & $8.59 \pm 0.66^{* *}$ & $6.57 \pm 0.34$ & $3.66 \pm 0.23^{* *}$ \\
\hline Final BW, kg & $26.33 \pm 0.81$ & $17.84 \pm 0.89^{*}$ & $46.86 \pm 3.25$ & $31.42 \pm 2.55^{* *}$ & $105.40 \pm 3.51$ & $81.71 \pm 3.23^{* *}$ \\
\hline $\mathrm{ADG}, \mathrm{kg} / \mathrm{d}$ & $0.59 \pm 0.01$ & $0.43 \pm 0.01^{*}$ & $0.65 \pm 0.15$ & $0.44 \pm 0.08^{*}$ & $0.69 \pm 0.08$ & $0.55 \pm 0.07^{* *}$ \\
\hline $\mathrm{ADFl}, \mathrm{kg} / \mathrm{d}$ & $0.96 \pm 0.04$ & $0.72 \pm 0.03^{* *}$ & $1.48 \pm 0.34$ & $0.92 \pm 0.12^{*}$ & $1.92 \pm 0.40$ & $1.48 \pm 0.26$ \\
\hline$F / G$ & $1.64 \pm 0.04$ & $1.67 \pm 0.08$ & $2.28 \pm 0.36$ & $2.09 \pm 0.16$ & $2.78 \pm 0.13$ & $2.71 \pm 0.22$ \\
\hline
\end{tabular}

Data are presented as means \pm SEM. The data presented are obtained from 12 animals in each group $(n=12)$. In the same row, values with * were significantly different from NBW pigs at $P<0.05$ and values with ** were significantly different at $P<0.01$. ADG, average daily gain; $A D F I$, average daily feed intake; $B W$, body weight; $F / G$, feed/gain; Initial BW, body weight at birth; NBW, normal born weight

\section{Plasma hormone profile}

Compared to NBW pigs, IUGR pigs in the $25 \mathrm{~kg}$ BW group had higher concentrations of plasma ghrelin, SS, PYY, and PP, but lower concentration of insulin $(P<$ $0.05)$. The IUGR pigs in the $50 \mathrm{~kg} B W$ group had higher concentrations of IGF-1, leptin, and insulin, but lower concentrations of gastrin, ghrelin, and PP $(P<0.05)$ than NBW pigs. No significant differences were observed between IUGR and NBW pigs in the $100 \mathrm{~kg}$ BW group (Table 3).

\section{S rRNA sequencing data}

A total of 4,237,106 high-quality DNA sequences were obtained from the high-throughput sequencing of 120 samples, which included 60 jejunum and 60 ileum samples from growing-finishing pigs in the 25, 50, and 100 kg BW groups. We randomly analyzed a subsample of 27,223 sequences for each sample, to avoid bias caused by different sequencing depths. The rarefaction curves obtained suggested that almost all bacterial species were captured using this approach (Supplementary Fig. 1).

\section{Microbiota diversity in the small intestine}

Differences in alpha diversity, including ACE (Fig. 1a), Simpson (Fig. 1b), Shannon (Fig. 1c), and Chao1 (Fig. 1d) indices, were assessed by performing the MannWhitney U-test. In the jejunum, IUGR pigs in the 25 kg BW group had significantly higher values of Simpson and Shannon indices than NBW pigs $(P<0.05)$; in the $100 \mathrm{~kg}$ BW group, ACE and Chao1 indices were also significantly higher in IUGR pigs. In the ileum, IUGR pigs in the $25 \mathrm{~kg}$ BW group had higher ACE $(P<0.01)$ and Chao1 $(P<0.05)$ indices than NBW pigs, but those in the $100 \mathrm{~kg} B W$ group had lower ACE $(P<0.05)$.

The beta-diversity analysis was performed to measure the dissimilarity of microbial communities between IUGR pigs and NBW pigs. PCoA plots did not indicate a clear separation between NBW pigs and IUGR pigs (Fig. 2). We thus performed a PLS-DA based on unweighted UniFrac distances. This approach revealed that microbial communities in the jejunum of NBW and IUGR pigs in the 25,50 , and $100 \mathrm{~kg} \mathrm{BW}$ groups and in the ileum of NBW and IUGR pigs in the 25 and $50 \mathrm{~kg}$

Table 3 Effect of intrauterine growth restriction (IUGR) on plasma hormones of growing-finishing pigs

\begin{tabular}{|c|c|c|c|c|c|c|}
\hline \multirow[t]{2}{*}{ Items } & \multicolumn{2}{|c|}{25 kg BW group } & \multicolumn{2}{|l|}{50 kg BW group } & \multicolumn{2}{|c|}{100 kg BW group } \\
\hline & NBW & IUGR & NBW & IUGR & NBW & IUGR \\
\hline Gastrin, ng/mL & $0.48 \pm 0.03$ & $0.50 \pm 0.02$ & $2.87 \pm 0.53$ & $1.68 \pm 0.18^{*}$ & $1.32 \pm 0.15$ & $1.49 \pm 0.09$ \\
\hline $\mathrm{GH}, \mathrm{ng} / \mathrm{mL}$ & $17.50 \pm 0.58$ & $18.27 \pm 0.63$ & $47.38 \pm 12.07$ & $32.24 \pm 4.43$ & $29.13 \pm 2.68$ & $30.05 \pm 2.05$ \\
\hline Ghrelin, ng/mL & $4.81 \pm 0.23$ & $5.81 \pm 0.26^{*}$ & $21.34 \pm 4.30$ & $11.19 \pm 1.30^{*}$ & $9.40 \pm 1.24$ & $10.62 \pm 0.67$ \\
\hline Glucagon, ng/mL & $0.32 \pm 0.01$ & $0.33 \pm 0.05$ & $0.52 \pm 0.13$ & $0.59 \pm 0.08$ & $0.40 \pm 0.04$ & $0.43 \pm 0.02$ \\
\hline IGF-1, ng/mL & $125.65 \pm 5.09$ & $140.27 \pm 6.12$ & $255.18 \pm 21.76$ & $399.38 \pm 60.88^{*}$ & $284.78 \pm 30.20$ & $269.82 \pm 12.57$ \\
\hline Insulin, mIU/L & $33.27 \pm 0.93$ & $29.72 \pm 1.14^{*}$ & $37.65 \pm 2.02$ & $46.61 \pm 3.42^{*}$ & $38.02 \pm 4.64$ & $37.52 \pm 4.15$ \\
\hline Leptin, ng/mL & $10.69 \pm 0.28$ & $12.08 \pm 0.74$ & $17.88 \pm 0.98$ & $29.38 \pm 4.94^{*}$ & $24.93 \pm 3.04$ & $22.99 \pm 1.82$ \\
\hline $\mathrm{PP}, \mathrm{ng} / \mathrm{mL}$ & $2.09 \pm 0.07$ & $2.65 \pm 0.10^{* *}$ & $12.01 \pm 1.50$ & $6.81 \pm 0.98^{* *}$ & $6.82 \pm 0.94$ & $7.69 \pm 0.70$ \\
\hline PYY, pmol/mL & $9.98 \pm 0.39$ & $14.91 \pm 0.61^{* *}$ & $28.54 \pm 6.65$ & $24.26 \pm 4.98$ & $19.83 \pm 2.08$ & $18.95 \pm 1.09$ \\
\hline $\mathrm{SS}, \mathrm{pg} / \mathrm{mL}$ & $89.11 \pm 3.17$ & $98.44 \pm 1.24^{*}$ & $193.49 \pm 59.58$ & $180.15 \pm 36.94$ & $145.80 \pm 15.03$ & $133.19 \pm 10.92$ \\
\hline
\end{tabular}

Data are presented as means \pm SEM. The data presented are obtained from 10 animals in each group $(n=10)$. In the same row, values with * were significantly different from NBW pigs at $P<0.05$ and values with ${ }^{* *}$ were significantly different at $P<0.01$. GH Growth hormone; IGF-1 Insulin-like growth factors-1, NBW Normal born weight, PP Pancreatic polypeptide, PYY Peptide YY, SS Somatostatin 

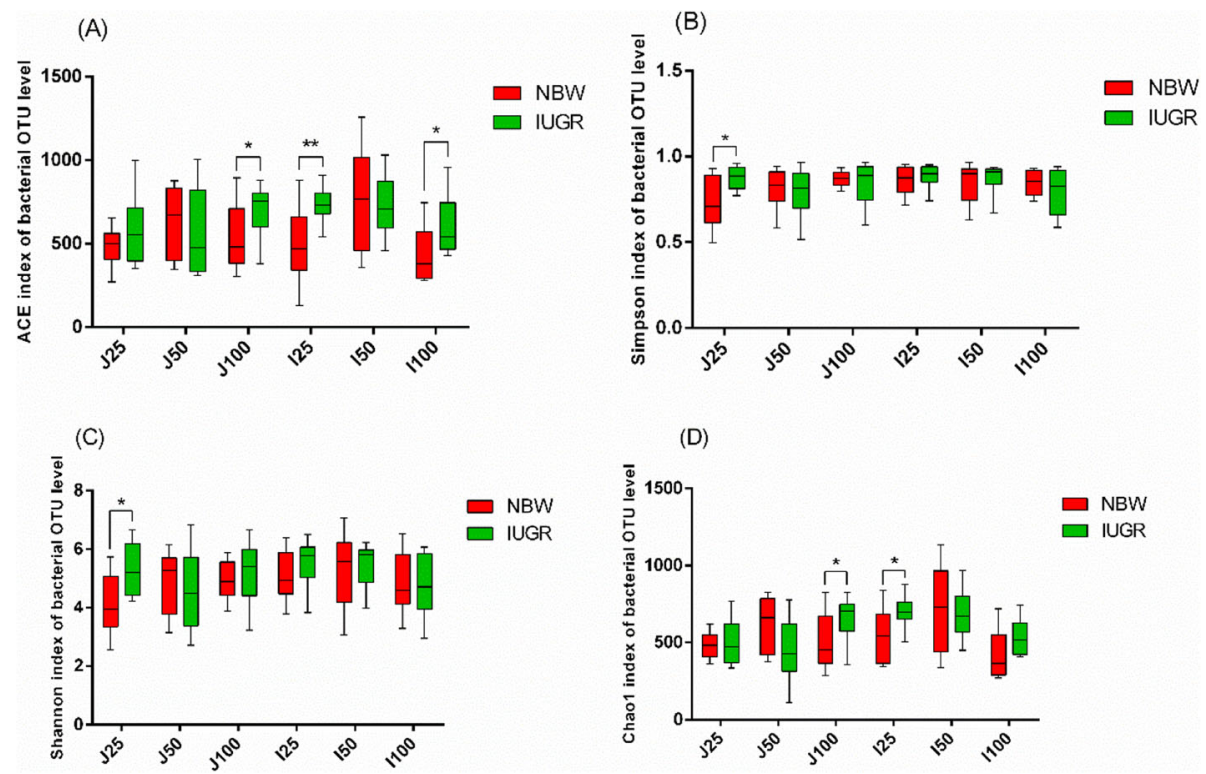

Fig. 1 Microbial alpha diversity in the jejunum and ileum contents of intrauterine growth retardation (IUGR) pigs and normal birth weight (NBW) pigs in the 25, 50, and $100 \mathrm{~kg}$ BW groups. The data presented are obtained from 10 animals in each group $(n=10)$. a ACE index, b Simpson index, c Shannon index, and $\mathbf{d}$ Chao 1 index. * denotes a significant difference $(P<0.05)$ among different treatments

BW groups were clearly separated and clustered into distinct groups.

\section{Microbial community composition in the small intestine}

Based on 97\% 16S rRNA gene sequence identity, we identified 19 microbial phyla and 221 microbial genera in the jejunum and 21 phyla and 223 genera in the ileum of IUGR and NBW pigs (Fig. 3).

Microbiota composition analysis showed that Firmicutes, Proteobacteria, Actinobacteria, and Bacteroidetes were the dominant microbial phyla in both IUGR pigs and NBW pigs in all BW groups. In the jejunum (Fig. 3a), Firmicutes, Proteobacteria, Actinobacteria, and Thermi were the dominant microbial phyla in NBW pigs of all BW groups, while Firmicutes, Proteobacteria, Actinobacteria, and Bacteroidetes were the most abundant in IUGR pigs. In the ileum (Fig. 3b), Firmicutes and Proteobacteria were the most abundant phyla, followed by Actinobacteria and Bacteroidetes in both IUGR pigs and NBW pigs. Other microbial phyla were present at very low relative abundances.

The abundance distribution in intestinal microbiota at the genus level was plotted as a heatmap (Fig. 3c). For IUGR pigs in the $25 \mathrm{~kg} \mathrm{BW}$ group, the four dominant genera in the jejunum were Cupriavidus (29.18\%), Streptococcus (12.87\%), Acinetobacter (6.14\%), and unclassified Clostridiaceae (4.66\%). In the $50 \mathrm{~kg} \mathrm{BW}$ group, the most abundant genera were Lactobacillus (73.63\%), unclassified Desulfovibrionaceae (6.87\%), Turicibacter (3.79\%), and unclassified Enterobacteriaceae (3.01\%). In the $100 \mathrm{~kg}$ BW group, Lactobacillus (68.46\%), Cupriavidus (12.80\%), Streptococcus (3.19\%), and Pseudomonas (2.44\%) were the most abundant genera. For NBW pigs, the dominant genera in the jejunum were Cupriavidus (46.35\%), Streptococcus (9.09\%), Acinetobacter (6.14\%), and unclassified Caulobacteraceae (3.91\%) in the $25 \mathrm{~kg}$ BW group; Cupriavidus (28.57\%), Lactobacillus (26.21\%), Streptococcus (6.57\%), and Pseudomonas (5.25\%) in the $50 \mathrm{~kg} \mathrm{BW}$ group; and Cupriavidus (22.54\%), Lactobacillus (20.64\%), Acinetobacter (8.15\%), and Pseudomonas (7.79\%) in the $100 \mathrm{~kg}$ BW group.

For IUGR pigs, the dominant genera in the ileum were Streptococcus (28.23\%), Lactobacillus (16.12\%), unclassified Clostridiaceae (12.03\%), and unclassified Ruminococcaceae (7.11\%) in the $25 \mathrm{~kg} \mathrm{BW}$ group; Lactobacillus (77.58\%), unclassified Clostridiaceae (4.65\%), Cupriavidus (4.31\%), and Streptococcus (3.08\%) in the $50 \mathrm{~kg} \mathrm{BW}$ group; and Lactobacillus (63.94\%), unclassified Enterobacteriaceae (9.51\%), unclassified Peptostreptococcaceae (9.40\%), and Turicibacter (4.69\%) in the $100 \mathrm{~kg} \mathrm{BW}$ group (Fig. 3d). For NBW pigs, the dominant genera in the ileum were unclassified Clostridiaceae (28.02\%), Streptococcus (15.09\%), Cupriavidus (9.11\%), and Actinobacillus (7.97\%) in the $25 \mathrm{~kg}$ BW group; Lactobacillus (35.15\%), Streptococcus (18.07\%), unclassified Peptostreptococcaceae (10.95\%), and Cupriavidus (9.75\%) in the $50 \mathrm{~kg}$ BW group; and Lactobacillus (26.42\%), unclassified Peptostreptococcaceae (22.27\%), Turicibacter (15.29\%), and unclassified Clostridiaceae (10.33\%) in the $100 \mathrm{~kg} \mathrm{BW}$ group. 


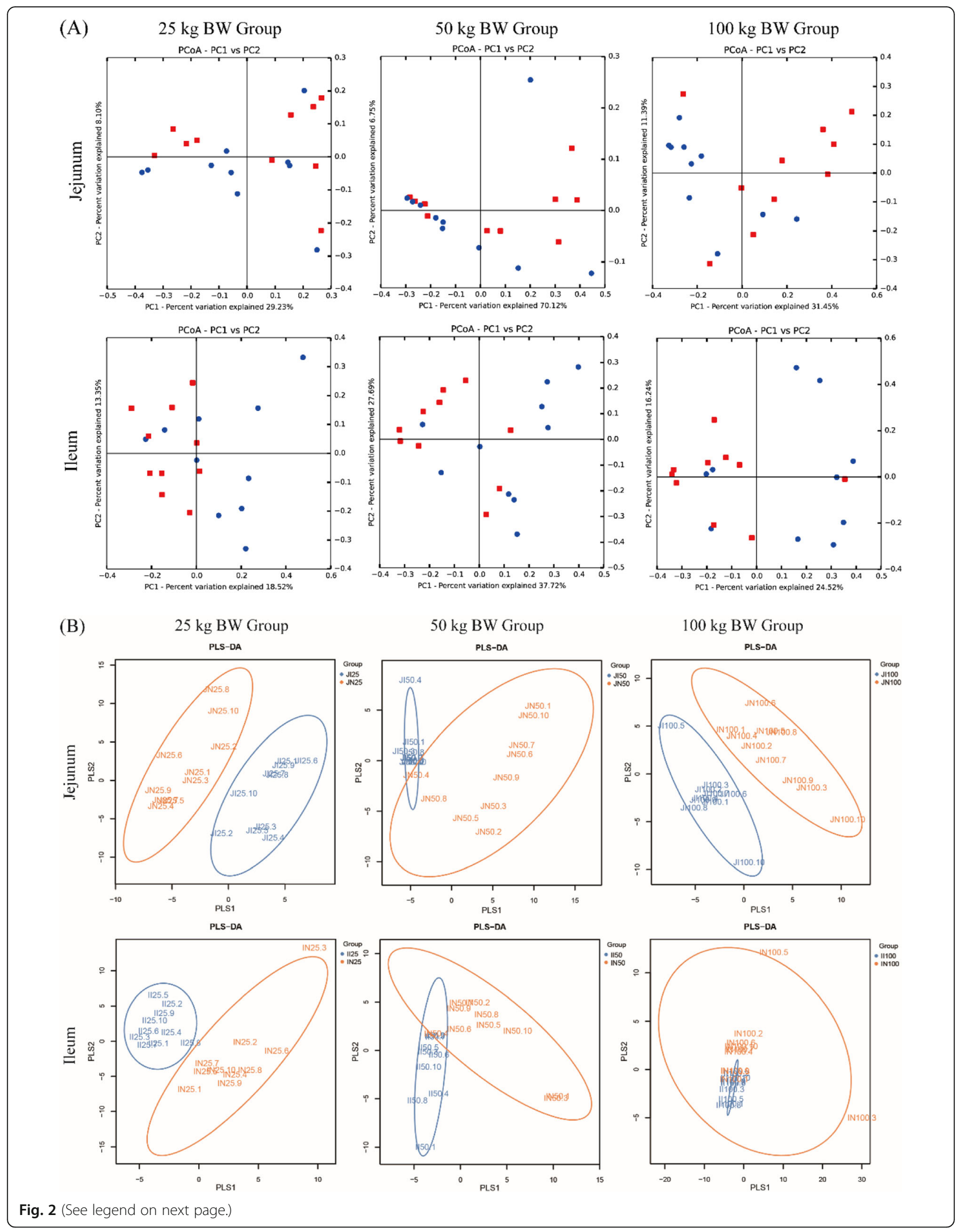


(See figure on previous page.)

Fig. 2 Differences in intestinal microbial community structure between intrauterine growth retardation

IUGR) pigs and normal birth weight ( NBW) pigs in the 25, 50, and $100 \mathrm{~kg}$ BW groups, and each symbol represents the intestinal microbiota of one pig. The data presented are obtained from 10 animals in each group $(n=10)$. a Principal coordinate analysis (PCoA) along the axes IUGR and NBW. b Partial least square discriminant analysis (PLS-DA) based on an unweighted UniFrac distances score plot of jejunum and ileum microbiota. Each point represents the microbial community of one pig

\section{Differences in the intestinal microbial communities of IUGR and NBW pigs}

Differences in the relative abundances of the community components of microbiota in the jejunum and ileum of NBW and IUGR pigs were further analyzed using the Mann-Whitney U-test by STAMP and LEfSe. Microbial phyla with relative abundance above $0.01 \%$ in the jejunum and in the ileum were selected for analysis (Fig. 4). Compared to NBW pigs, IUGR pigs in the $25 \mathrm{~kg} \mathrm{BW}$ group had a higher abundance of Bacteroidetes in the ileum $(P<0.05)$, and those in the $50 \mathrm{~kg} B W$ group had higher abundance of Firmicutes $(P<0.05)$ and lower abundances of Proteobacteria, Thermi, Cyanobacteria, and Bacteroidetes in the jejunum $(P<0.05)$. In the 100 kg BW group, IUGR pigs had a higher abundance of Firmicutes $(P<0.05)$ and lower abundances of Fusobacteria, Proteobacteria, and Thermi in the jejunum $(P<0.05)$, as compared to NBW pigs. We observed no significant differences in the relative abundance of microbial phyla in the jejunum of pigs in the $25 \mathrm{~kg}$ BW group and in the ileum of pigs in the 50 and $100 \mathrm{~kg}$ BW groups.

At the genus level, the top 20 most abundant taxa in the combined samples were analyzed using LEfSe to identify differences, defined as an LDA score above 3.0 (Fig. 5). In the $25 \mathrm{~kg} \mathrm{BW}$ group, Amycolatopsis and Ochrobactrum were significantly less abundant in the jejunum of IUGR pigs than in the jejunum of NBW pigs $(P<0.05)$. In the $50 \mathrm{~kg} B W$ group, IUGR pigs had significantly higher abundance of Lactobacillus in the jejunum but significantly lower abundances of unclassified Ruminococcaceae, Cupriavidus, unclassified Pseudomonadaceae, Pseudomonas, unclassified Lachnospiraceae, Ochrobactrum, unclassified Erysipelotrichaceae, Sphingomonas, unclassified Caulobacteraceae, Acinetobacter, Thermus, unclassified Clostridiales, and unclassified YS2 than NBW pigs $(P<$ 0.05). In the $100 \mathrm{~kg} B W$ group, IUGR pigs had significantly higher abundance of Lactobacillus in the jejunum but significantly lower abundances of Cupriavidus, Thermus, Acinetobacter, Sphingomonas, unclassified Clostridiales, Amycolatopsis, unclassified Clostridiaceae, and unclassified Peptostreptococcaceae than NBW pigs $(P<0.05)$. In the 25 $\mathrm{kg}$ BW group, the abundances of Lactobacillus, unclassified Ruminococcaceae, and Parabacteroides in the ileum of IUGR pigs was significantly higher $(P<0.05)$, while that of Actinobacillus was significantly lower, than in NBW pigs $(P<0.05)$. In the $50 \mathrm{~kg}$ BW group, Lactobacillus and unclassified Streptophyta had significantly higher abundance in the ileum of IUGR pigs $(P<0.05)$, while Streptococcus, unclassified Peptostreptococcaceae, SMB53, unclassified Mogibacteriaceae, Turicibacter, unclassified Clostridiales, and unclassified Erysipelotrichaceae had significantly lower abundance than in NBW pigs $(P<0.05)$. In the $100 \mathrm{~kg} \mathrm{BW}$ group, Lactobacillus and unclassified Streptococcaceae had higher abundance in the ileum of IUGR pigs, while unclassified Clostridiales had lower abundance, than in NBW pigs $(P<0.05)$.

\section{Correlation between intestinal microbial community composition and growth performance of pigs}

To analyze the relationship between intestinal microbiota composition and growth performance of growingfinishing pigs, Spearman's correlations were calculated between the microbial relative abundance of the jejunum and ileum (based on the top 20 abundant taxa at the genus level) and ADG, initial BW, weaned BW, and final BW of IUGR pigs and NBW pigs in the 25, 50, and 100 kg BW groups (Fig. 6).

In the jejunum, Lactobacillus was negatively correlated with growth performance. However, unclassified Peptostreptococcaceae, Thermus, Cupriavidus, Sphingomonas, unclassified Clostridiales, unclassified Ruminococcaceae, and unclassified Clostridiaceae were positively correlated with the initial and weaned BW in the 50 (Fig. 6b) and $100 \mathrm{~kg}$ BW (Fig. 6c) groups; Ochrobactrum and Amycolatopsis were positively correlated with the initial BW in the 25 (Fig. 6a) and $50 \mathrm{~kg}$ BW groups; and Gemella, unclassified Caulobacteraceae, unclassified Comamonadaceae, Pseudomonas, unclassified Pseudomonadaceae, Acinetobacter, and Streptococcus were positively correlated with the growth performance in the $50 \mathrm{~kg} \mathrm{BW}$ group.

In the ileum, Lactobacillus was negatively correlated with initial BW as well as weaned BW in the $25 \mathrm{~kg}$ BW (Fig. 6d) group and with growth performance only in the $50 \mathrm{~kg}$ BW (Fig. 6e) group. On the contrary, unclassified Ruminococcaceae and unclassified Clostridiales were positively correlated with the growth performance in the 50 and $100 \mathrm{~kg} \mathrm{BW}$ (Fig. 6f) groups; unclassified Lachnospiraceae was positively correlated with the ADG and final BW in the $25 \mathrm{~kg}$ BW group; and unclassified Enterobacteriaceae, unclassified Peptostreptococcaceae, and Streptococcus were positively correlated with the growth performance in the $50 \mathrm{~kg} \mathrm{BW}$ group. 


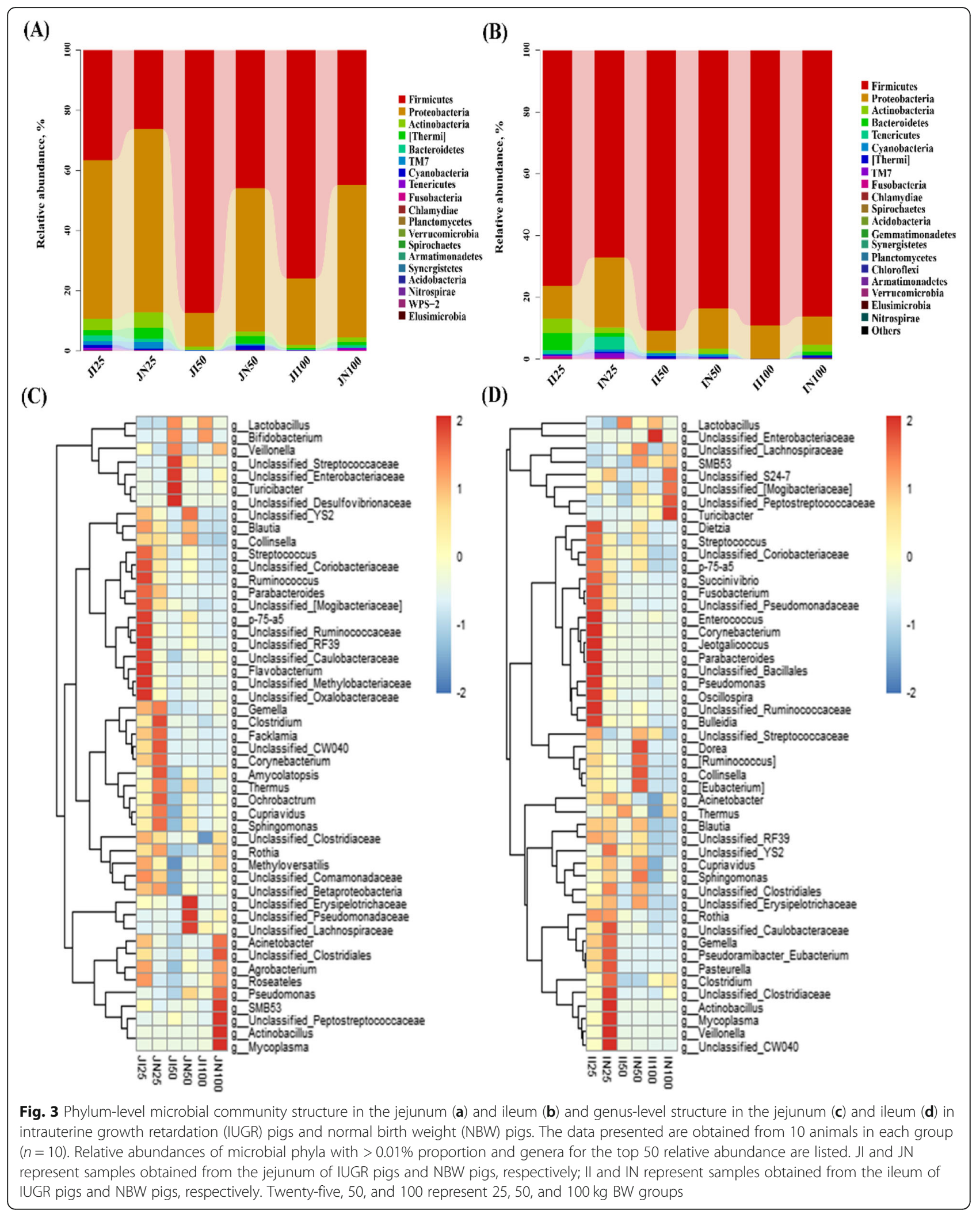




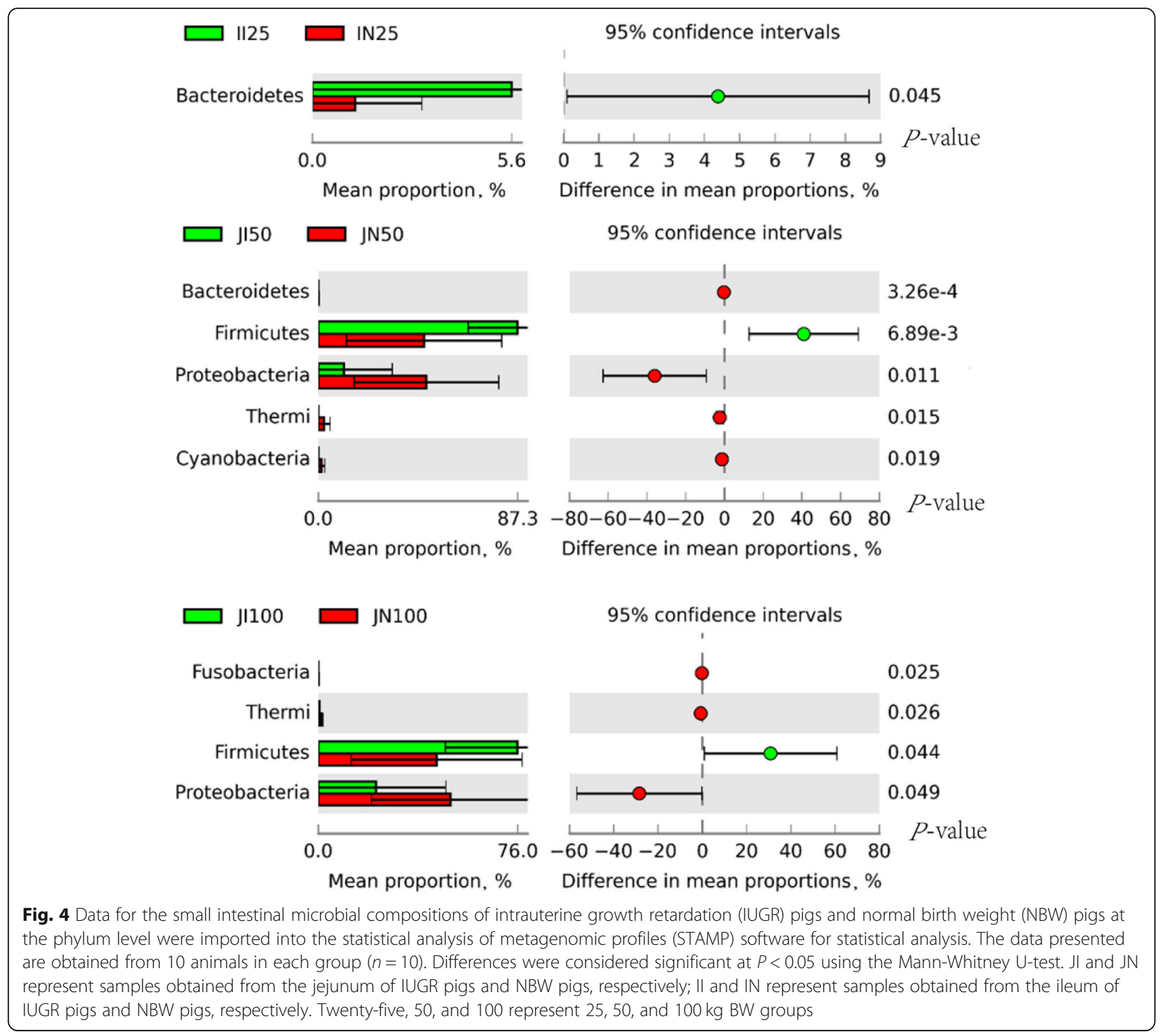

Metabolic capability profiles of small intestine microbiota PICRUSt analysis showed that metabolic pathways were classified into six functional categories (level 1) and used to compare the functional enrichment in IUGR pigs and NBW pigs (Fig. 7a). Moreover, we performed the same comparison by using 45 different gene functions (level 2) (Fig. 7b). Metabolism was the predominant functional pathway affected by the intestinal microbiota in both IUGR pigs and NBW pigs. However, the relative enrichment of different metabolic pathways in the intestinal microbiota appears to allow the discrimination between IUGR pigs and NBW pigs. LEfSe analysis was then performed to observe differences in gene functions (level 3; Fig. 8). Because many significantly enriched functional pathways were common between the 50 and $100 \mathrm{~kg}$ BW groups, we subsequently focused on these pathways in the jejunum and ileum of IUGR and NBW pigs.
In the jejunum, the expression of genes in the following categories was enriched in IUGR pigs compared to that in NBW pigs in the 50 and $100 \mathrm{~kg} \mathrm{BW}$ groups (LDA > 3.0, $P<0.05)$ : ion-coupled transporters, starch and sucrose metabolism, DNA replication, peptidoglycan biosynthesis, mismatch repair, homologous recombination, chromosome, galactose metabolism, phosphotransferase system, glycolysis/gluconeogenesis, purine metabolism, peptidases, DNA replication proteins, aminoacyl-t RNA biosynthesis, amino sugar and nucleotide sugar metabolism, pyrimidine metabolism, DNA repair and recombination proteins, ribosome function, and ribosome biogenesis. Conversely, the expression of genes in the following categories was enriched in NBW pigs compared to that in IUGR pigs in the 50 and $100 \mathrm{~kg}$ BW groups (LDA > 3.0, $P<0.05$ ): bacterial motility proteins, two-component systems, degradation or metabolism of various amino acids, fatty acid 

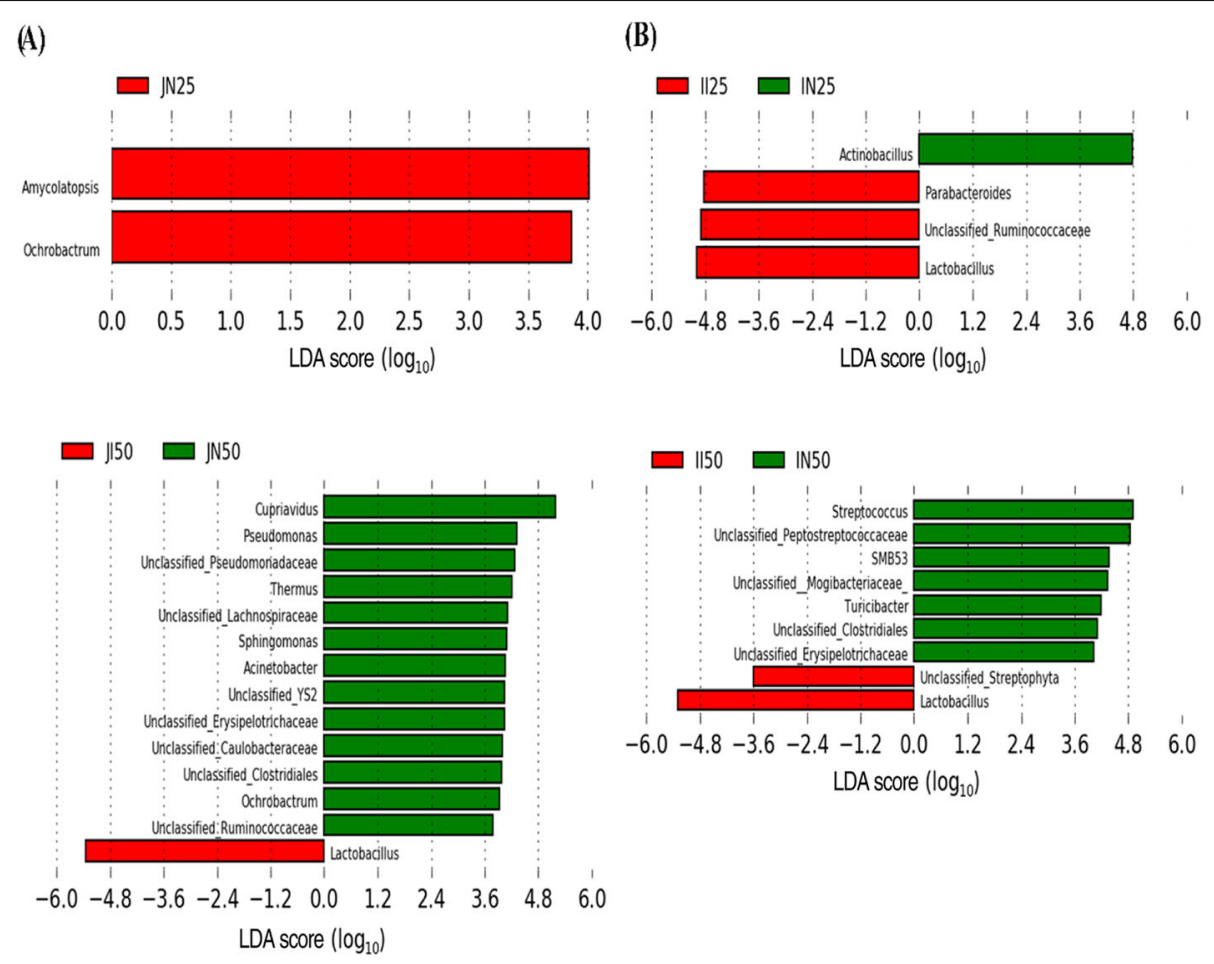

$$
6.0
$$
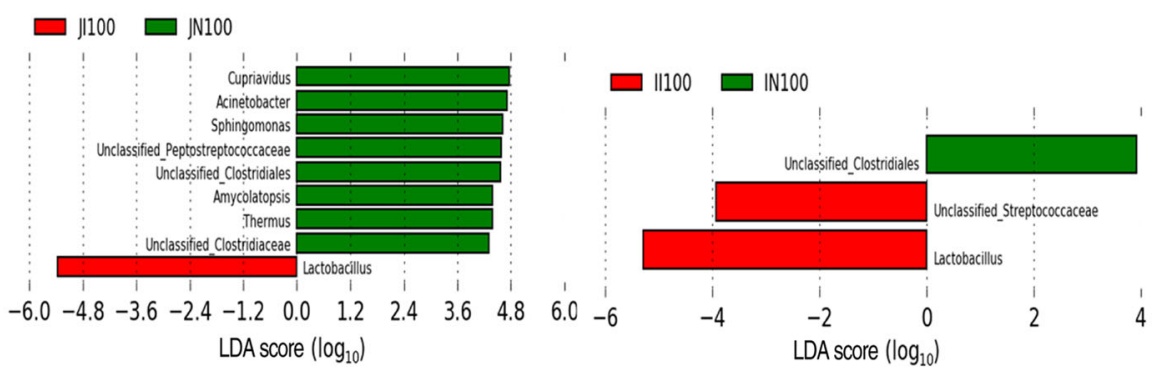

Fig. 5 LEfSe analysis of small intestinal microbial compositions of intrauterine growth retardation (IUGR) pigs and normal birth weight (NBW) pigs throughout the trial at genus level. The data presented are obtained from 10 animals in each group $(n=10)$. Significant differences (LDA score $\geq$ $3, P<0.05)$ for jejunum and ileum in growing-finishing pigs are shown. JI and JN represent samples obtained from the jejunum of IUGR pigs and NBW pigs, respectively; II and IN represent samples obtained from the ileum of IUGR pigs and NBW pigs, respectively. Twenty-five, 50, and 100 represent 25, 50, and $100 \mathrm{~kg}$ BW groups

metabolism, porphyrin and chlorophyll metabolism, betaalanine metabolism, glyoxylate and dicarboxylate metabolism, propanoate metabolism, flagellar assembly, benzoate degradation, butanoate metabolism, geraniol degradation, and limonene and pinene degradation (Fig. 8).

In the ileum, the expression of genes involved in ioncoupled transporters, purine metabolism, and replication, recombination, and repair proteins was enriched in IUGR pigs compared to that in NBW pigs in the 50 and $100 \mathrm{~kg} \mathrm{BW}$ groups (LDA $>3.0, P<0.05$ ). The ABC transporter pathway was enriched in NBW pigs compared to that in IUGR pigs in the 25 and $50 \mathrm{~kg} \mathrm{BW}$ groups, and phenylalanine, tyrosine, and tryptophan biosynthesis and energy metabolism pathways were enriched in NBW pigs compared to that in IUGR pigs in the 25 and $100 \mathrm{~kg}$ BW groups (Fig. 9).

\section{Discussion}

In a previous study, we reported alterations in the microbial communities of the small intestine in IUGR piglets at 7, 21, and $28 \mathrm{~d}$ of age, and that Bacteroidetes, Bacteroides, Escherichia-Shigella, and Pasteurella could be related to nutrient digestion and absorption, and growth and development regulation [14]. Microbial communities in the intestine are influenced by various environmental factors, including age, diet, lifestyle, and medication [17]. However, whether intestinal microbiota colonization during lactation has an effect during the 


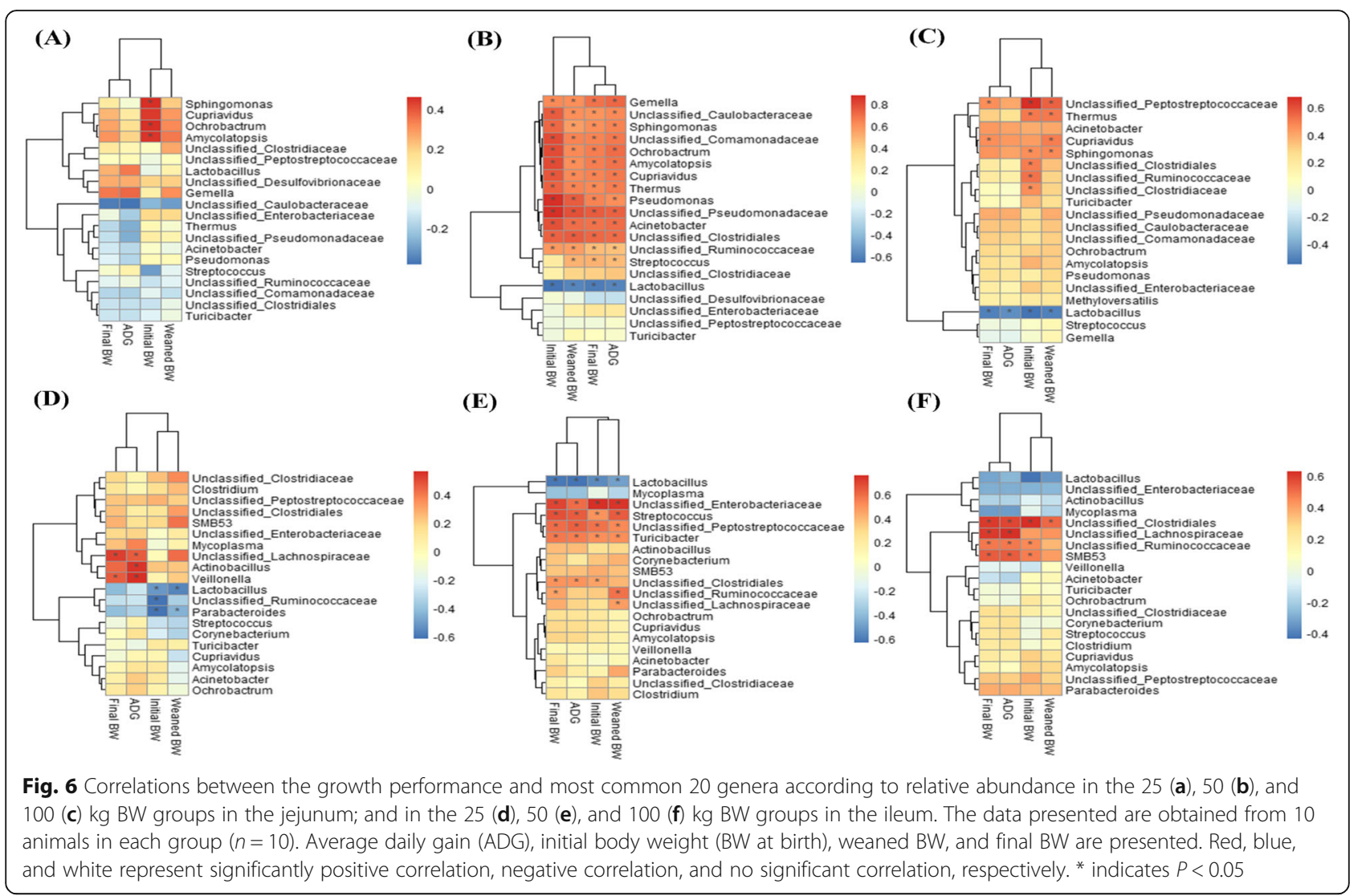

growing-finishing period in IUGR pigs remains unclear. Thus, in the present study, we further analyzed the effects of IUGR on the microbial communities in the small intestine and plasma hormone profiles in growingfinishing pigs in the 25,50 , and $100 \mathrm{~kg} \mathrm{BW}$ groups. Our study clearly showed that IUGR is associated with impaired growth after weaning, which is concomitant with alterations in the hormonal profile, intestinal microbiota alpha diversity, and microbiota composition.

Growth performance is usually greater when pigs are penned individually than penned in groups [18]. The reported factors related to reductions in ADFI and ADG in group-penned pigs include competition and aggressive behavior to maintain dominance hierarchy, an increase standing, and physiological responses due to chronic stress of weaning, competition, and aggressive encounters [19]. To reduce the influence of these factors, the pigs were individually penned in the present study. Specifically, IUGR piglets had significantly lower initial BW, weaned $B W$, final BW, ADG, and ADFI compared to NBW pigs in the 25,50 , and $100 \mathrm{~kg} \mathrm{BW}$ groups, consistent with previous results $[20,21]$. The decrease in ADG might have resulted from the reduced abundance of unclassified Peptostreptococcaceae, unclassified Clostridiales, unclassified Clostridiaceae, unclassified Pseudomonadaceae, and
Pseudomonas, which are positively correlated with ADG. These results confirmed the known effects of IUGR on pig growth, as low birth weight leads to decreased postnatal growth rate.

Insulin promotes the synthesis of glycogen, fat, and protein [22]. The lower plasma insulin levels observed in IUGR pigs in the $25 \mathrm{~kg}$ BW group were consistent with those found in previous studies [23]. IUGR impairs both the exocrine and endocrine pancreatic development and reduces pancreatic weight in pigs [24], thereby concomitantly reducing plasma insulin levels [25]. However, the increase in plasma insulin in the $50 \mathrm{~kg}$ BW group may suggest that pancreatic impairment was restored during growth, in a manner similar to the observed increase in feed efficiency, and maybe also as the result of an adaptive process. Ghrelin is a $\mathrm{GH}$-stimulating hormone, produced and secreted primarily from the stomach [26]. PP is a polypeptide hormone released by the pancreas, which suppresses appetite and food intake [27]. We found that IUGR pigs had increased plasma ghrelin and PP levels compared to NBW pigs in the $25 \mathrm{~kg}$ BW group, but these levels decreased in the $50 \mathrm{~kg} \mathrm{BW}$ group, indicating that the kinetics of hormone secretion in IUGR pigs during growth is complex. A possible rationale for this result may be that the plasma hormone 
(A)

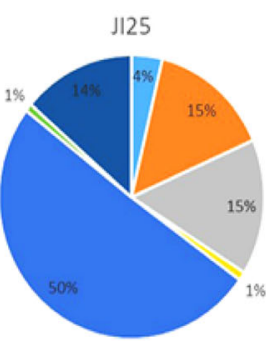

$J 150_{2 \%}$

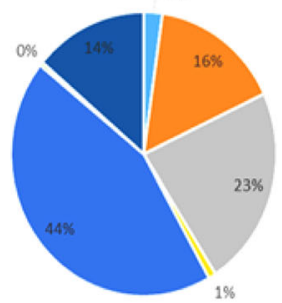

J100

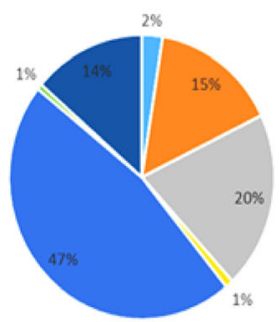

- Cellular Processes

- Metabolism

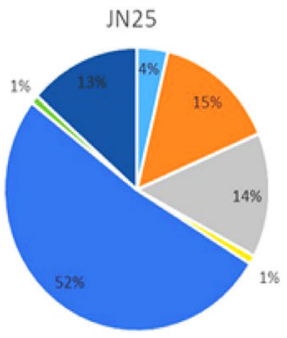

JN50

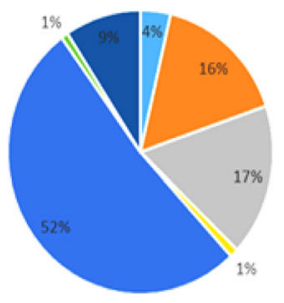

JN100

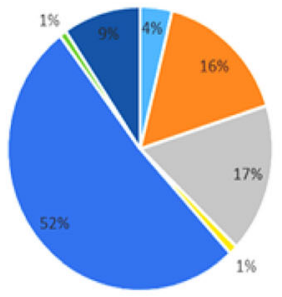

- Environmental Information Processing

- Organismal System

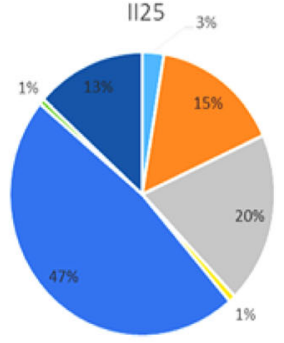

1150

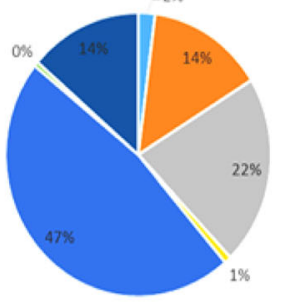

$11100 \quad 2 \%$

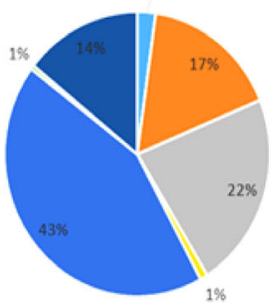

II Genetic Information Processing

- Unclassified

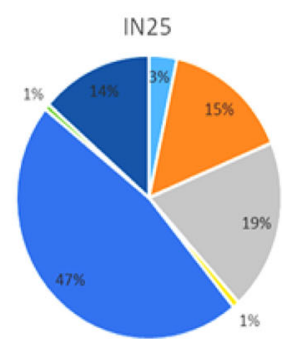

IN50

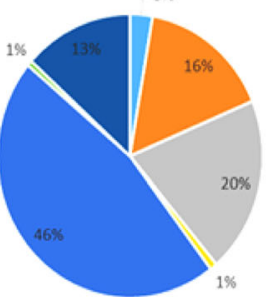

IN100

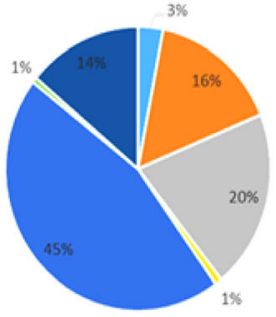

Human Diseases

(B)

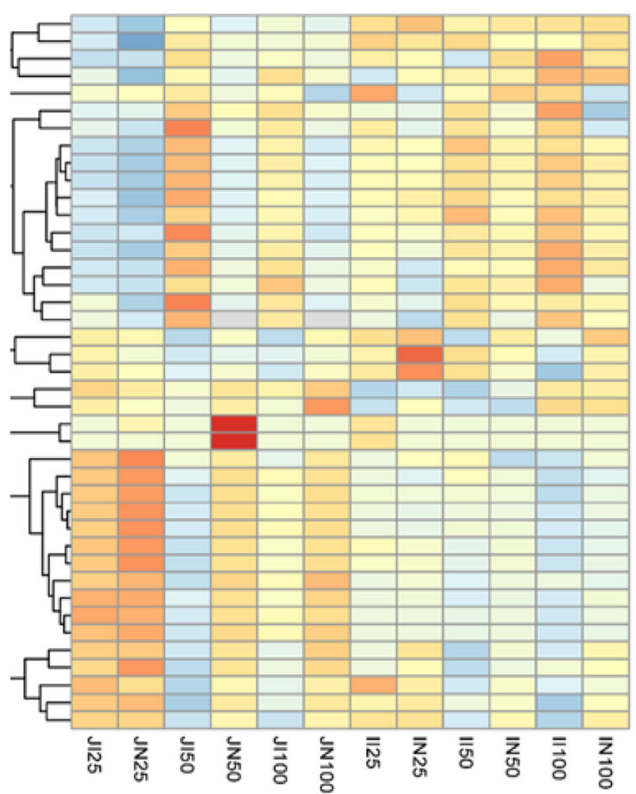

Genetic Information Processing(level 1): (level 2) Transcription

Organismal Systems(level 1); (level 2) Nervous System
Environmental Information Processing(level 1): (level 2) Membrane Transport

Organismal Systems(level 1); (level 2) Environmental Adaptation

Metabolism(level 1); (level 2) Carbohydrate Metabolism

Human Diseases(level 1); (level 2) Infectious Diseases
Metabolism(level 1): (level 2) Glycan Biosynthesis and Metabolism

Metabolism(level 1) (level 2) Nucleotide Metabolism
Genetic Information Processing(level 1); (level 2) Replication and Repair Genetic Information Processing(level 1): (level 2) Translation Genetic Information Processing (level 1); (level 2) Folding, Sor Human Diseases(level 1); (level 2) Metabolic Diseases

Metabolism(level 1); (level 2) Enzyme Families

Environmental Information Processing(level 1); (level 2) Signaling Molecules and Interaction Human Diseases(level 1); (level 2) Immune System Diseases
Cellular Processes(level i) (level 2) Cell Growth and Death

Unclassified(level 1): (leve', 2) Poorly Characterized

Unclassical (evel 1). (level 2) Poorly Characterized

Metabolism(level 1); (level 2) Energy Metabolism

Human Diseases(level 1); (level 2) Cancers
Unclassified(level 1); (level' 2) Cellular Processes and Signaling

Cellular Processes (level 1): (level 2) Cell Communication

Organismal Systems (level 1); (level 2) Sensory System

Organismal Systems (level 1) (level 2) Excretory System
Metabolism(level 1): (level 2 Metabolism of Terpenoids and Polyketides

Metabolism(level 1) (leve 22 Metabolasm of Other Amino Acids

Cellular Processes (level 1); (level 2) Transport and Catabolism

Organismal Systems(level 1); (level 2) Endocrine System

nvironmental Information Processing(level 1); (level 2 ) Signal Tra
Human Diseases(level 1): (level 2) Neurodegenerative Diseases

Human Diseases(level 1): (level 2) Cardiovascular Diseases
Hel

Organismal Systems(level 1); (level 2) Circulatory System

Cellular Processes(level 1); (level 2) Cell Motility

Unclassified(level 1); (level 2) Metabolism
Organismal Systems (level 1); (level 2) Digestive System

etabolism(level 1): (level 2) Biosynthesis of Other Secondary Metabolites

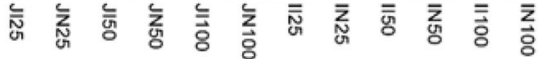

Fig. 7 (See legend on next page.) 
(See figure on previous page.)

Fig. 7 Predictive metagenomics showing differences in function between intrauterine growth retardation (IUGR) pigs and normal birth weight (NBW) pigs in the jejunum and ileum using PICRUSt analysis at level 1 (a) and level 2 (b). The data presented are obtained from 10 animals in each group $(n=10)$. JI and JN represent samples obtained from the jejunum of IUGR pigs and NBW pigs, respectively; II and IN represent samples obtained from the ileum of IUGR pigs and NBW pigs, respectively. Twenty-five, 50, and 100 represent 25, 50, and $100 \mathrm{~kg}$ BW groups

profiles may represent the early situation before plasma sampling, and that individual factors in IUGR and NBW pigs may lead to alterations in such profiles.

Intestinal microbiota alpha diversity has been associated with host health and metabolic capacity [28]. In pigs, intestinal bacteria diversity changes from weaning to growing and finishing stages [29]. We observed that alpha diversity of the jejunum and ileum microbiota in IUGR pigs was significantly higher than in NBW pigs in all BW groups evaluated, suggesting that IUGR pigs had a more diversified intestinal microbiota. A recent study showed that IUGR pigs had significantly higher alpha diversity ( $\mathrm{ACE}$ and Chaol indices) in the ileum than NBW pigs at $70 \mathrm{~d}$ of age [30]. The abundance of adherent
(A)

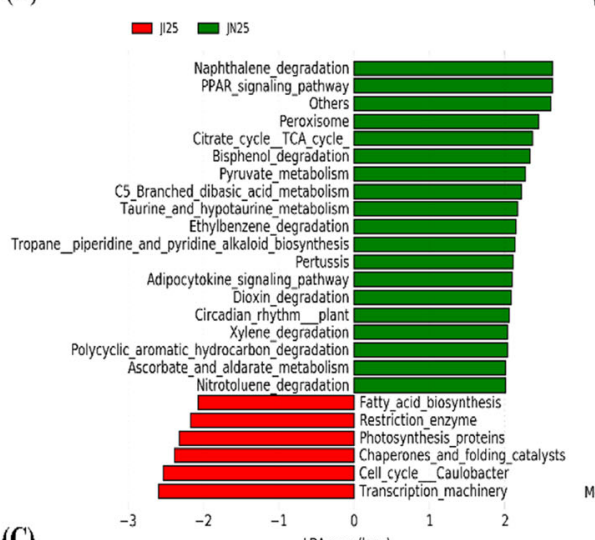

(C)

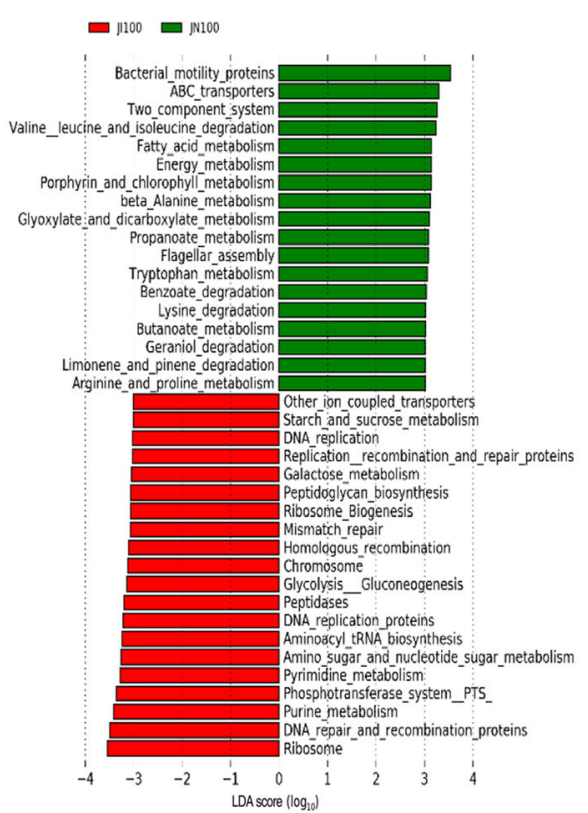

(B)

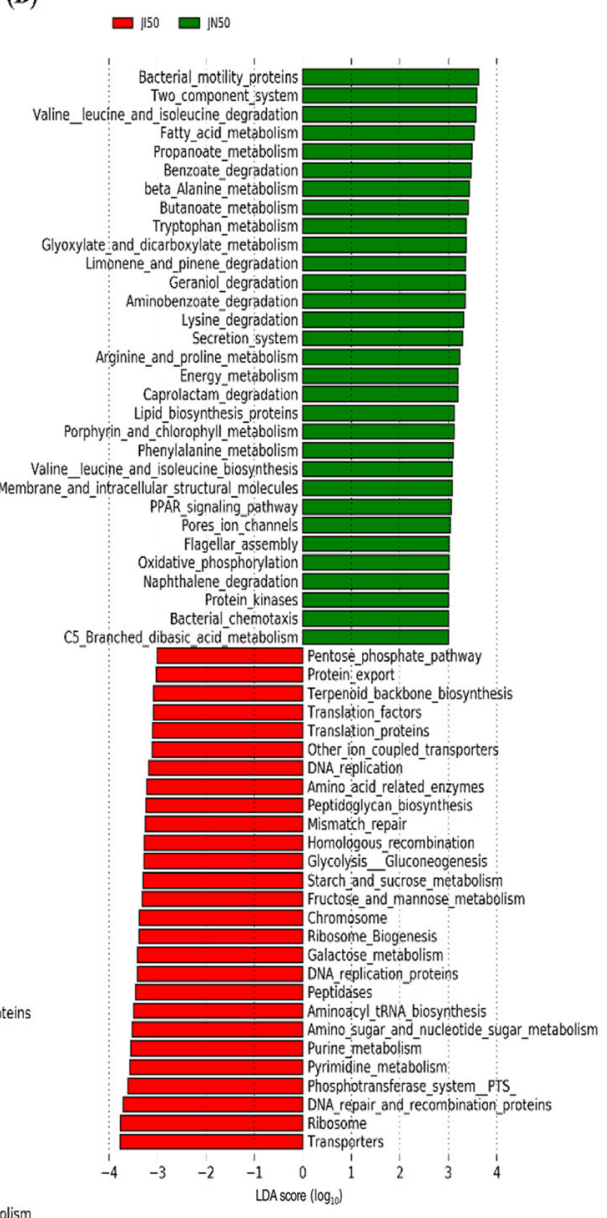

Fig. 8 Predictive metagenomics showing differences in function between intrauterine growth retardation (IUGR) and normal birth weight (NBW) pigs in the jejunum using PICRUSt analysis (level 3). The data presented are obtained from 10 animals in each group $(n=10)$. J and JN represent samples obtained from the jejunum of IUGR pigs and NBW pigs, respectively; 25, 50, and 100 represent 25, 50, and $100 \mathrm{~kg}$ BW groups. LEfSe analysis was utilized to identify potentially enriched pathways. Pathways with LDA scores $\geq 2$ are shown in the $25 \mathrm{~kg}$ BW group (a), and pathways with LDA scores $\geq 3$ in the 50 (b) and 100 (c) $\mathrm{kg}$ BW groups 


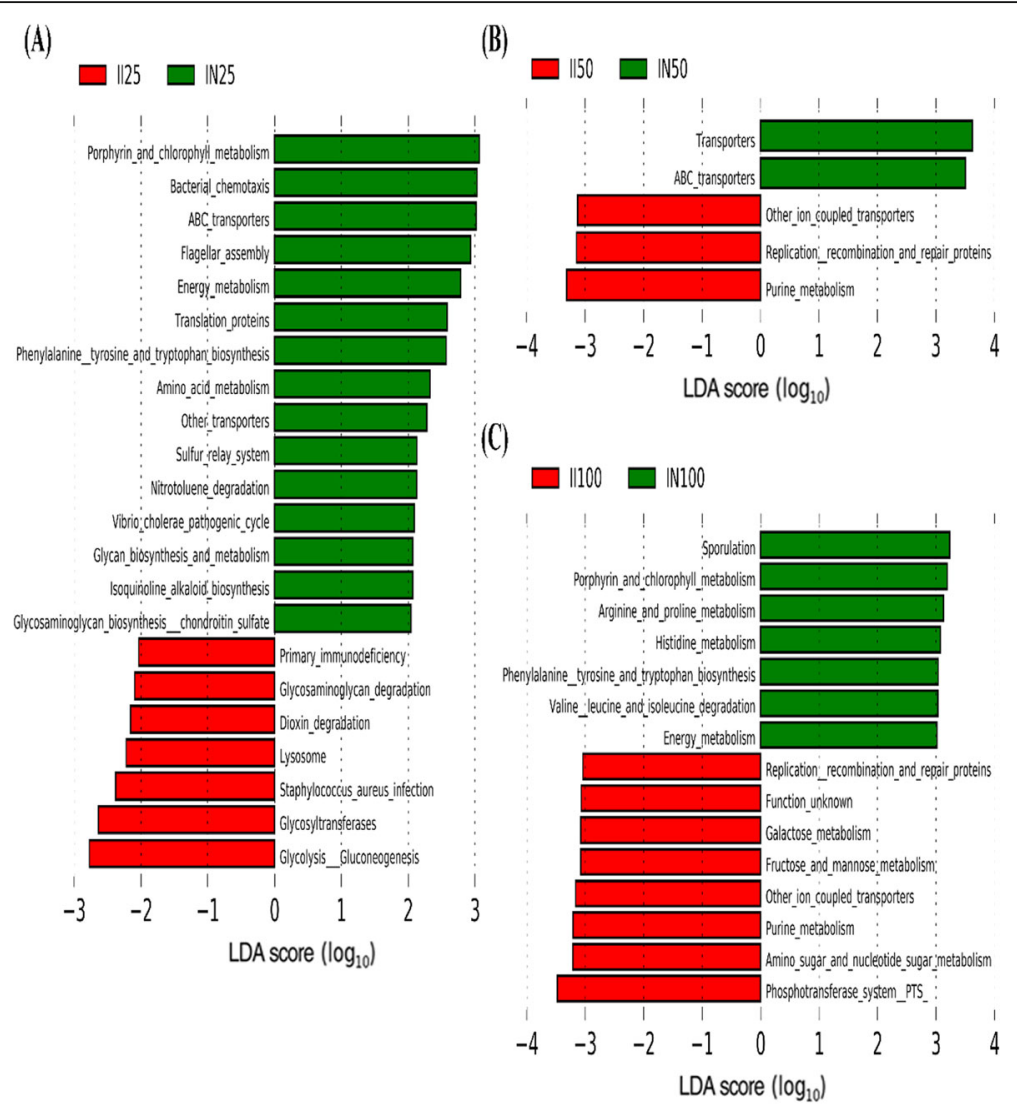

Fig. 9 Predictive metagenomics showing differences in function between intrauterine growth retardation (IUGR) pigs and normal birth weight (NBW) pigs in the ileum using PICRUSt analysis (level 3). The data presented are obtained from 10 animals in each group $(n=10)$. II and IN represent samples obtained from the ileum of IUGR pigs and NBW pigs, respectively. Twenty-five, 50, and 100 represent 25, 50, and 100 kg BW. LEfSe analysis was used to identify potentially enriched pathways. Pathways with LDA scores $\geq 2$ in the $25 \mathrm{~kg}$ BW group (a), and LDA scores $\geq 3$ in the 50 (b) and 100 (c) kg BW groups are shown

bacteria in the intestinal mucosa of IUGR piglets has been shown to be higher than that in NBW piglets [31]. Accordingly, in the present study, the beta-diversity analysis showed that IUGR significantly altered the microbial community in the small intestine.

Firmicutes and Proteobacteria were the two most abundant microbial phyla detected in the ileum of both NBW pigs and IUGR pigs, in agreement with a previous study [32]. Firmicutes is associated with energy absorption from nutrition [33], while members of the Bacteroidetes phylum are specialized in the degradation of proteins and carbohydrates [34]. In the present study, the higher relative abundance of Firmicutes in IUGR pigs in the 50 and $100 \mathrm{~kg} \mathrm{BW}$ groups, which was consistent with the results of a previous study [35], suggested that the intestinal microbiota of IUGR pigs may be more efficient than NBW pigs at recovering energy from the diet, notably for covering the high energy need of intestinal epithelium cell, although this needs to be validated experimentally. The Proteobacteria phylum includes a wide variety of pathogens (such as Escherichia,
Salmonella, and Yersinia), and members of this phylum serve as indicators of intestinal inflammation and epithelial dysfunction [36]. Although IUGR pigs in the 50 and $100 \mathrm{~kg}$ BW groups showed a lower proportion of Proteobacteria in the jejunum compared to NBW pigs, their growth performance remained restricted. This phenomenon may be associated with impaired intestinal morphology and gastrointestinal dysfunction in IUGR pigs [8].

Within the Firmicutes phylum, Lactobacillus has been identified as a key genus in the intestine of pigs [37], with proportions ranging from $0.53-4.51 \%$ of the total ileal bacteria and $4.98-17.84 \%$ of the colonic bacteria in finishing pigs [38]. Lactobacillus utilizes fermentative carbohydrates and produces lactic acid as a major end product, thereby supplying energy to the cells of the intestinal epithelium [39]. In our study, IUGR pigs in the 50 and $100 \mathrm{~kg}$ BW groups had a significantly higher abundance of Lactobacillus in the small intestine than NBW pigs, but Lactobacillus abundance in the jejunum and ileum was negatively correlated with the growth 
performance. Lactobacillus is taxonomically complex and composed of over 170 species, 17 subspecies of which are occasional opportunistic pathogens [40]. For instance, $L$. rhamnosus and L. rhamnosus $G G$ may be at the origin of Lactobacillus bacteremia [41]. These opposite roles of Lactobacillus species make it difficult to define clearly classify them as probiotics or pathogens for IUGR pigs. As a producer of short-chain fatty acids (SCFAs), the Ruminococcaceae family is responsible for the degradation of diverse polysaccharides and fibers. The production of SCFAs is considered beneficial for the maintenance of intestinal health [42]. Our findings showed that unclassified genera belonging to the Ruminococcaceae were the most abundant taxa in both IUGR pigs and NBW pigs. In the ileum of IUGR pigs in the 25 $\mathrm{kg}$ BW group, Ruminococcaceae showed higher abundance than in the ileum of NBW pigs during the growing period, in accordance with a recent study [30]. Dietary carbohydrates could be efficiently fermented by Ruminococcaceae during the growing stage, thereby enabling the bioavailability of luminal energy substrates. However, in a recent study, the mRNA expression of SCFA transporters (FFAR1, FFAR2, and PPAR) and their absorption capacity were decreased in IUGR pigs during the growth period [30]. Thus, the higher amounts of SCFAs fermented by Ruminococcaceae might not be totally absorbed and utilized by the intestinal epithelial cells effectively, which leads to nutrient loss in IUGR pigs during the growing period. Ochrobactrum is a genus belonging to the Proteobacteria phylum containing 15 species [43], of which $O$. tritic and O. intermedium are emerging pathogens capable of causing infections in healthy individuals [44]. We found that IUGR pigs in the 25 and $50 \mathrm{~kg}$ BW groups had a lower abundance of Ochrobactrum in the jejunum than NBW pigs. However, if such a low abundance of Ochrobactrum in IUGR piglets confers a beneficial effect with respect to infection remains to be determined.

In the present study, the abundance of several bacteria, such as Firmicutes, Ruminococcaceae, and Lactobacillus, was increased in the jejunum and ileum, which may lead to an increased production of SCFAs and lactic acid to supply extra energy to the intestinal epithelial cells of IUGR pigs. This phenomenon could be explained with the "thrifty phenotype" hypothesis, which suggests that when nutritional conditions in the uterus are suboptimal, the growth and metabolism of the fetus are restricted. However, when the postnatal nutritional condition is adequate, IUGR pigs undergo a catch-up growth with respect to the intestinal microbiota [45]. This phenotype is presumably the result of an adaptation to adverse conditions. However, the energy supply through microbial fermentation from luminal substrates remains an inefficient method of digesting nutrients and may lead to an imbalanced nutrient supply in IUGR pigs [30]. Due to the multifaceted effect of impaired organ development, intestinal morphology, insufficient maternal colostrum intake, and Lactobacillus bacteremia, among others [46], the energy from microbial fermentation is insufficient to prevent growth delay, thereby leading to restricted growth performance during the growing-finishing period of IUGR pigs. Despite the growth compensation by beneficial intestinal microbiota colonization in IUGR pigs when a high quality diet was provided, a lower return in production performance over investment value was still observed for these pigs.

In the present study, PICRUSt analysis showed that IUGR altered several microbial gene functions. Carbohydrate and amino acid metabolism were the dominant functions in both IUGR pigs and NBW pigs, likely because the main ingredients of the feed and substrate type of predominant microbial fermentation are carbohydrates and proteins. Interestingly, intestinal microbiota of IUGR pigs displayed, in terms of gene expression, a presumably higher capacity for carbohydrate metabolism, translation, nucleotide metabolism, galactose metabolism, glycan biosynthesis and metabolism, DNA replication and repair, and cellular processes and signaling. The higher expression in IUGR pigs of genes involved in carbohydrate metabolism and glycan biosynthesis and metabolism was associated with a higher proportion of Firmicutes and Lactobacillus. Lactobacillus use fermentative carbohydrates to produce lactic acid [39], while Firmicutes can metabolize proteins and carbohydrates to produce SCFAs [35]. Moreover, we found that the expression of genes involved in DNA replication, mismatch repair, chromosome and homologous recombination, and ribosome biogenesis was upregulated in the jejunum microbiota of IUGR pigs in the 50 and $100 \mathrm{~kg}$ BW groups, suggesting that IUGR might regulate gene expression in the intestinal bacteria, in agreement with the results of a previous study [32].

Interestingly, when compared to NBW pigs, IUGR pigs showed lower expression of genes involved in lipid metabolism, glyoxylate and dicarboxylate metabolism, amino acid metabolism, energy metabolism, terpenoid and polyketide metabolism, xenobiotic biodegradation and metabolism, cofactor metabolism, and vitamin metabolism, consistent with the results of our previous study [14]. The downregulation of amino acid metabolism is consistent with lower concentrations of most amino acids observed in IUGR pigs [47]. Because of this, dietary supplementation with functional amino acids (e.g. arginine, tryptophan, and lysine) may help to mitigate growth restriction and improve the health and growth of IUGR pigs $[48,49]$.

Further studies are needed to determine the possible causal links between the changes observed in the intestinal microbiota and the changes in the measured 
physiological parameters. Consistent with this hypothesis, different bacterial metabolites have been shown to modify the secretion of various hormones produced by the enteroendocrine cells of the intestinal epithelium [50]. Moreover, lower feed intake may have an impact on nutrient availability, due to the metabolic activity of the intestinal microbiota [51].

\section{Conclusion}

In this study, IUGR pigs presented a lower growth performance, higher microbial alpha diversity, and different plasma concentrations of hormones during the growingfinishing period, as compared to NBW pigs. Alterations in the abundances of Firmicutes, Proteobacteria, Ruminococcaceae, Lactobacillus, and Ochrobactrum in the small intestine may be associated with IUGR, and could therefore serve as a future target for intestinal microbiota interventions in growing-finishing pigs subjected to IUGR.

\section{Supplementary information}

Supplementary information accompanies this paper at https://doi.org/10 1186/s40104-020-00490-x

Additional file 1: Figure S1. Differences in microbial community structures in the jejunum (A) and ileum (B) between intrauterine growth retardation (IUGR) pigs and normal birth weight (NBW) pigs throughout the trial. The data presented are obtained from 10 animals in each group $(n=10)$. Rarefaction curve analysis was used to evaluate whether further sequencing would likely detect additional taxa. JI and JN represent samples obtained from the jejunum of IUGR pigs and NBW pigs, respectively; II and IN represent samples obtained from the ileum of IUGR pigs and NBW pigs, respectively. 25, 50, and 100 represent 25, 50, and $100 \mathrm{~kg}$ BW groups.

\section{Abbreviations}

BW: Body weight; IUGR: Intrauterine growth restriction; LEfSe: Linear discriminant analysis effect size; NBW: Normal birth weight; OTU: Operational taxonomic units; SCFA: Short-chain fatty acid

\section{Acknowledgments}

We thank technicians from CAS Key Laboratory of Agro-ecological Processes in Subtropical Region for providing technical assistance and the Hunan New WellFul Co., Ltd. in Hunan Province, China, for their support in the pig experiments. We would like to thank Editage (www.editage.cn) for English language editing.

\section{Authors' contributions}

$\mathrm{XL}$ and $\mathrm{ZWH}$ performed the animal feeding experiment and samples analysis. $X \mathrm{~L}, \mathrm{YJM}, \mathrm{KXF}$, and FB wrote the manuscript. KXF and $Y Y L$ contributed to experimental concepts and design, provided scientific direction, and finalized the manuscript. All authors read and approved the final manuscript.

\section{Funding}

This present study was jointly supported by the National Natural Science Foundation of China (31772613 and 31572421) and Special Funds for Construction of Innovative Provinces in Hunan Province (2019RS3022).

\section{Availability of data and materials}

The data generated or analyzed during the current study are available from the corresponding author by reasonable request.

\section{Ethics approval and consent to participate}

All animal protocols used in this study were in accordance with the Chinese Guidelines for Animal Welfare and approved by the Animal Care and Use Committee of Institute of Subtropical Agriculture, the Chinese Academy of Sciences.

\section{Consent for publication}

Not applicable.

\section{Competing interests}

The authors declare that no competing interests exist. The manuscript has not been published previously.

\section{Author details}

${ }^{1}$ CAS Key Laboratory of Agro-ecological Processes in Subtropical Regions, Hunan Provincial Key Laboratory of Animal Nutritional Physiology and Metabolic Process, National Engineering Laboratory for Pollution Control and Waste Utilization in Livestock and Poultry Production, Institute of Subtropical Agriculture, Chinese Academy of Sciences, Changsha 410125, Hunan, China. ${ }^{2}$ Key Laboratory of Animal Nutrition in Jiangxi Province, College of Animal Science and Technology, Jiangxi Agricultural University, Nanchang 440000, Jiangxi, China. ${ }^{3}$ Université Paris-Saclay, AgroParisTech, INRAE, UMR PNCA, 75005 Paris, France.

Received: 5 March 2020 Accepted: 23 June 2020

Published online: 19 August 2020

\section{References}

1. Wu G, Bazer FW, Cudd TA, Meininger CJ, Spencer TE. Maternal nutrition and fetal development. J Nutr. 2004;134:2169-72.

2. Pallotto EK, Kilbride HW. Perinatal outcome and later implications of intrauterine growth restriction. Clin Obstet Gynecol. 2006;49:257-69.

3. Wu G, Bazer FW, Wallace JM, Spencer TE. Board-invited review: intrauterine growth retardation: implications for the animal sciences. J Anim Sci. 2006; 84:2316-37.

4. Aw TY. Intestinal glutathione: determinant of mucosal peroxide transport, metabolism, and oxidative susceptibility. Toxicol Appl Pharmacol. 2005;204: 320-8.

5. Xu RJ, Mellor DJ, Birtles MJ, Reynolds GW, Simpson HV. Impact of intrauterine growth retardation on the gastrointestinal tract and the pancreas in newborn pigs. J Pediatr Gastroenterol Nutr. 1994;18:231-40.

6. Wang J, Chen L, Li D, Yin Y, Wang X, Li P, et al. Intrauterine growth restriction affects the proteomes of the small intestine, liver, and skeletal muscle in newborn pigs. J Nutr. 2008;138:60-6.

7. Zhang LL, Zhang H, Li Y, Wang T. Effects of medium-chain triglycerides on intestinal morphology and energy metabolism of intrauterine growth retarded weanling piglets. Arch Anim Nutr. 2017;71:231-45.

8. Hu L, Peng X, Chen H, Yan C, Liu Y, Xu Q, et al. Effects of intrauterine growth retardation and Bacillus subtilis PB6 supplementation on growth performance, intestinal development and immune function of piglets during the suckling period. Eur J Nutr. 2017;56:1753-65.

9. Buffie CG, Pamer EG. Microbiota-mediated colonization resistance against intestinal pathogens. Nat Rev Immunol. 2013;13:790-01.

10. Kamada N, Seo SU, Chen GY, Nunez G. Role of the gut microbiota in immunity and inflammatory disease. Nat Rev Immunol. 2013;13:321-35.

11. Blachier F, Beaumont M, Andriamihaja M, Davila AM, Lan A, Grauso M, et al. Changes in the luminal environment of the colonic epithelial cells and physiopathological consequences. Am J Pathol. 2017;187:476-86.

12. Kilby MD, Verhaeg J, Gittoes N, Somerset DA, Clark PM, Franklyn JA Circulating thyroid hormone concentrations and placental thyroid hormone receptor expression in normal human pregnancy and pregnancy complicated by intrauterine growth restriction (IUGR). J Clin Endocrinol Metab. 1998:83:2964-71

13. Fattal-Valevski A, Toledano-Alhadef H, Golander A, Leitner Y, Harel S. Endocrine profile of children with intrauterine growth retardation. J Pediatr Endocrinol Metab. 2005;18:671-6.

14. Zhang W, Ma C, Xie P, Zhu Q, Wang X, Yin Y, et al. Gut microbiota of newborn piglets with intrauterine growth restriction have lower diversity and different taxonomic abundances. J Appl Microbiol. 2019;127:354-69.

15. McArdle $\mathrm{BH}$, Anderson MJ. Fitting multivariate models to community data: a comment on distance-based redundancy analysis. Ecology. 2001;82:290-7. 
16. Segata N, Izard J, Waldron L, Gevers D, Miropolsky L, Garrett WS, et al. Metagenomic biomarker discovery and explanation. Genome Biol. 2011;12:R60.

17. Sommer F, Bäckhed F. The gut microbiota--masters of host development and physiology. Nat Rev Microbiol. 2013;11:227-38.

18. Hacker RR, Du Z, D'arcy CJ. Influence of penning type and feeding level on sexual behavior and feet and leg soundness in boars. J Anim Sci. 1994;72: 2531-7

19. Gomez RS, Lewis AJ, Miller PS, Chen HY. Growth performance and digestive and metabolic responses of gilts penned individually or in groups of four. J Anim Sci. 2000;78:597-603.

20. Beaulieu AD, Aalhus JL, Williams NH, Patience JF. Impact of piglet birth weight, birth order, and litter size on subsequent growth performance, carcass quality, muscle composition, and eating quality of pork. J Anim Sci. 2010;88:2767-78.

21. Rehfeldt C, Tuchscherer A, Hartung M, Kuhn G. A second look at the influence of birth weight on carcass and meat quality in pigs. Meat Sci. 2008:78:170-5

22. Efstratiadis A. Genetics of mouse growth. Int J Dev Biol. 1998;42:955-76.

23. Harada E, Shizuyama M, Ihara N, Takeuchi T. Impaired pancreatic endocrine and exocrine responses in growth-retarded piglets. J Vet Med A. 2003;50: 433-41.

24. Wang Y, Zhang L, Zhou G, Liao Z, Ahmad H, Liu W, et al. Dietary L-arginine supplementation improves the intestinal development through increasing mucosal Akt and mammalian target of rapamycin signals in intra-uterine growth retarded piglets. Brit J Nutr. 2012;108:1371-81.

25. Ogata ES, Swanson SL, Collins JW Jr, Finley SL. Intrauterine growth retardation: altered hepatic energy and redox states in the fetal rat. Pediatr Res. 1990;27:56-63.

26. Kojima M, Hosoda H, Date Y, Nakazato M, Matsuo H, Kangawa K. Ghrelin is a growth-hormone-releasing acylated peptide from stomach. Nature. 1999; 402:656-60.

27. Thieme V, Jolly N, Madsen AN, Bellmann-Sickert K, Schwartz TW, Holst B, et al. High molecular weight PEGylation of human pancreatic polypeptide at position 22 improves stability and reduces food intake in mice. $\mathrm{Br} \mathrm{J}$ Pharmacol. 2016;173:3208-21.

28. Clarke SF, Murphy EF, O'Sullivan O, Lucey AJ, Humphreys M, Hogan A, et al. Exercise and associated dietary extremes impact on gut microbial diversity. Gut. 2014:63:1913-20.

29. Kim HB, Borewicz K, White BA, Singer RS, Sreevatsan S, Tu ZJ, et al. Longitudinal investigation of the age-related bacterial diversity in the feces of commercial pigs. Vet Microbiol. 2011;153:124-33.

30. Huang SM, Wu ZH, Li TT, Liu C, Han DD, Tao SY, et al. Perturbation of the lipid metabolism and intestinal inflammation in growing pigs with low birth weight is associated with the alterations of gut microbiota. Sci Total Environ. 2020;719:137382.

31. D'Inca R, Kloareg M, Gras-Le Guen C, Le Huerou-Luron I. Intrauterine growth restriction modifies the developmental pattern of intestinal structure, transcriptomic profile, and bacterial colonization in neonatal pigs. J Nutr. 2010;140:925-31

32. Li N, Huang S, Jiang L, Dai Z, Li T, Han D, et al. Characterization of the early life microbiota development and predominant Lactobacillus species at distinct gut segments of low- and normal-birth-weight piglets. Front Microbiol. 2019:10:797.

33. Turnbaugh PJ, Hamady M, Yatsunenko T, Cantarel BL, Duncan A, Ley RE, et al. A core gut microbiome in obese and lean twins. Nature. 2009;457: 480-4.

34. Thomas F, Hehemann JH, Rebuffet E, Czjzek M, Michel G. Environmental and gut bacteroidetes: the food connection. Front Microbiol. 2011;2:93.

35. Matheson SM, Walling GA, Edwards SA. Genetic selection against intrauterine growth retardation in piglets: a problem at the piglet level with a solution at the sow level. Genet Sel Evol. 2018;50:46.

36. Litvak Y, Byndloss MX, Tsolis RM, Baumler AJ. Dysbiotic Proteobacteria expansion: a microbial signature of epithelial dysfunction. Curr Opin Microbiol. 2017;39:1-6.

37. Valeriano VD, Balolong MP, Kang DK. Probiotic roles of Lactobacillus sp. in swine: insights from gut microbiota. J Appl Microbiol. 2017;122:554-67.

38. Fan $\mathrm{P}$, Liu P, Song $\mathrm{P}$, Chen $\mathrm{X}, \mathrm{Ma} X$. Moderate dietary protein restriction alters the composition of gut microbiota and improves ileal barrier function in adult pig model. Sci Rep. 2017;7:43412.

39. O' Callaghan J, O' Toole PW. Lactobacillus: host-microbe relationships. Curr Top Microbiol Immunol. 2013;358:119-24.
40. Goldstein EJ, Tyrrell KL, Citron DM. Lactobacillus species: taxonomic complexity and controversial susceptibilities. Clin Infect Dis. 2015;60:S98S107.

41. Salminen MK, Rautelin H, Tynkkynen S, Poussa T, Saxelin M, Valtonen V, et al. Lactobacillus bacteremia, clinical significance, and patient outcome, with special focus on probiotic L. rhamnosus GG. Clin Infect Dis. 2004;38:62-9.

42. Hooda S, Boler BMV, Serao MCR, Brulc JM, Staeger MA, Boileau TW, et al. 454 pyrosequencing reveals a shift in fecal microbiota of healthy adult men consuming polydextrose or soluble corn fiber. J Nutr. 2012;142:1259-65.

43. Wi YM, Peck KR. Biliary sepsis caused by Ochrobactrum anthropi. Jpn J Infect Dis. 2010;63:444-6.

44. Thoma B, Straube E, Scholz HC, Al Dahouk S, Zoller L, Pfeffer M, et al. Identification and antimicrobial susceptibilities of Ochrobactrum spp. Int J Med Microbiol. 2009;299:209-20.

45. Nevin CL, Formosa E, Maki Y, Matushewski B, Regnault TRH, Richardson BS Maternal nutrient restriction in Guinea pigs as an animal model for studying growth-restricted offspring with postnatal catch-up growth. Am J Physiol Regul Integr Comp Physiol. 2018;314:R647-R54.

46. Vergauwen H, Degroote J, Prims S, Wang W, Fransen E, Smet SD, et al. Artificial rearing influences the morphology, permeability and redox state of the gastrointestinal tract of low and normal birth weight piglets. J Anim Sci Biotechnol. 2017:8:30.

47. Lin G, Liu C, Feng C, Fan Z, Dai Z, Lai C, et al. Metabolomic analysis reveals differences in umbilical vein plasma metabolites between normal and growth-restricted fetal pigs during late gestation. J Nutr. 2012;142:990-8.

48. Wu G. Functional amino acids in nutrition and health. Amino Acids. 2013;45: 407-11.

49. Lin G, Wang $X$, Wu G, Feng $C$, Zhou H, Li D, et al. Improving amino acid nutrition to prevent intrauterine growth restriction in mammals. Amino Acids. 2014;46:1605-23.

50. Portune KJ, Beaumont M, Davila AM, Tomé D, Blachier F, Sanz Y. Gut microbiota role in dietary protein metabolism and health-related outcomes: the two sides of the coin. Trends Food Sci Tech. 2016:57:213-32.

51. Lan A, Blachier F, Benamouzig R, Beaumont M, Barrat C, Coelho D, et al. Mucosal healing in inflammatory bowel diseases: is there a place for nutritional supplementation? Inflamm Bowel Dis. 2015;21:198-207.

\section{Ready to submit your research? Choose BMC and benefit from:}

- fast, convenient online submission

- thorough peer review by experienced researchers in your field

- rapid publication on acceptance

- support for research data, including large and complex data types

- gold Open Access which fosters wider collaboration and increased citations

- maximum visibility for your research: over $100 \mathrm{M}$ website views per year

At $\mathrm{BMC}$, research is always in progress.

Learn more biomedcentral.com/submissions 